\title{
Algebraic Aspects of Two-Dimensional Convolutional Codes
}

\author{
Ettore Fornasini and Maria Elena Valcher
}

\begin{abstract}
Two-dimensional (2-D) codes are introduced as linear shift-invariant spaces of admissible signals on the discrete plane. Convolutional and, in particular, basic codes are characterized both in terms of their internal properties and by means of their input-output representations. The algebraic structure of the class of all encoders that correspond to a given convolutional code is investigated and the possibility of obtaining 2-D decoders, free from catastrophic errors, as well as efficient syndrome decoders is considered. Some aspects of the state space implementation of 2-D encoders and decoders via (finite memory) 2-D system are discussed.
\end{abstract}

Index Terms-Encoders and decoders of 2-D sequences, dual codes, behaviors, 2-D state models, inverse systems.

\section{INTRODUCTION}

$\mathrm{T}$ HE algebraic theory of one-dimensional (1-D) convolutional codes was originated by a noteworthy series of papers G. D. Forney, Jr., published in the early 1970's [1]-[3]. Employing the same polynomial matrix techniques utilized in research on multivariable linear systems, Forney laid on firm foundations the notions of equivalence, minimality, and duality of convolutional encoders and showed how one could apply the state-space realization methods for implementing a code in a transmission chain.

Recently, the extension of the above techniques to polynomial matrices in two variables [4]-[7], guaranteed a fairly good understanding of their algebraic properties and made possible two significant advances in 2-D signal modeling and realization, which seem very promising for applications in multidimensional data coding.

The first such development is the behavioral approach, introduced by J. C. Willems and P. Rocha [8]-[11] in the description of the admissible 2-D system trajectories. This approach, indeed, allows one to investigate the recursive structure of the codes without making any a priori assumption on the direction of the recursion and, consequently, on the specific kind of causality to which the encoding process refers. Moreover, once a convolutional code $\mathscr{E}$ has been selected on the basis of some internal requirements (such as the reliability of the transmitted message, the distance between two distinct codewords etc.), it is possible to provide a complete description of all

Manuscript received January 11, 1993; revised October 22, 1993.

The authors are with the Department of Electronics and Computer Science, University of Padova, 35131 Padova, Italy.

IEEE Log Number 9403850
Laurent polynomial encoders that produce $\mathscr{E}$, and to find among them the most efficient ones.

The second major development is the introduction of 2-D finite memory systems [12]-[14], which constitute the natural state-model for realizing polynomial transfer matrices in two indeterminates and, therefore, for implementing a code using digital hardware.

Seeking to make a contribution to the evolutionary trend described above, this paper outlines an algebraic theory of 2-D convolutional codes, which encompasses both a behavioral approach to the internal structure of the codes and a state-space procedure for synthesizing 2-D encoders and decoders. For these reasons, Willems theory constitutes the natural framework of our treatment, and the results of $P$. Rocha [9] provide a natural format for the analysis of many distinguishing features of 2-D convolutional codes.

In the first portion of the paper, 2-D convolutional codes are introduced as modules of doubly indexed sequences. Several connections with the submodule of finite codewords are discussed, thus providing different characterizations of the convolutional property and a complete classification of all equivalent encoders.

The next part deals with 2-D basic codes and injective encoders. Unlike the 1-D case, a 2-D convolutional code needs not admit an injective encoder. So "good" codes constitute only a proper subclass of the convolutional ones, and characterizing such a class requires the introduction of the notions of extendability and left zero-prime encoders.

Most of the concepts introduced in the previous parts are revisited in the section devoted to the notion of duality. The different point of view adopted therein finds a very natural application in the synthesis of 2-D syndrome decoders.

In the last section we concentrate on some aspects of the realization problem, considering finite memory 2-D systems as candidates for its solution. The quarter plane causality that underlies the state updating of these models requires the introduction of some restrictions on the support of the information signals to be encoded, and one must cope with standard polynomials, instead of Laurent polynomials, in representing encoders and decoders. Finally, in order to reduce the computational effort involved in designing the transmission chain, we investigate the possibility of realizing 2-D decoders as inverse state models of the corresponding encoders. 
Due to the intrinsic complexity of the subject, some results still have a preliminary character and some topics remain rather unexplored. Nevertheless, it is hoped that the main features of the theory have been covered, and some directions for future developments are broadly visible from our exposition. In particular, looking to the future, a detailed analysis of the distance properties of 2-D behaviors would constitute an important goal in the subsequent development of the theory. As error-detecting and error-correcting features are likely related to the distance between the codewords, these properties provide important criteria when 2-D behaviors are used as errorcorrecting codes on a noisy channel. On the other hand, when considering the implementation of 2-D encoders and decoders, it would clearly be desirable to relate the properties of a 2-D polynomial matrix to the dimension of its minimal state-space realizations. This could eventually lead to an expression of the necessary conditions for obtaining an optimal encoder for a given code $\mathscr{E}$ as a constraint on the polynomial structure of the encoder itself. At the present time, however, little is known concerning the structure of 2-D minimal realizations and, as a consequence, there is no way to single out, among the equivalent encoders of a given 2-D code, those that exhibit the most economical realizations.

\section{2-D CONVOLUTIONAL CODES AND THEIR ENCODERS}

A 2-D code $\mathscr{C}$ of length $n$ over a finite field $F$ can be viewed as a set of sequences indexed on the discrete plane $\mathbf{Z} \times \mathbf{Z}$ and taking values in $\mathbf{F}^{n}$. Thus, denoting the sequence space $\left(\mathbf{F}^{n}\right)^{\mathbf{Z} \times \mathbf{Z}}$ as $\mathscr{F}_{\infty}^{n}$, it follows that $\mathscr{E}$ is a subset of $\mathscr{F}_{\infty}^{n}$.

In 1-D coding theory, the natural order of $Z$ is usually associated with the time ordering and, therefore, with the sequential structure of the data flow. This motivates the habit of considering 1-D codewords with left compact support, and to represent them [15] as vectors with components in the field $F((z))$ of formal power series with left compact support.

When encoding two-dimensional data, there is no natural notion of causality inducing a particular ordering in $\mathbf{Z} \times \mathbf{Z}$ and, consequently, some a priori restrictions on the supports of the sequences in $\mathscr{C}$. So, adopting this point of view, we will, in general, assume that the supports of the elements of the code could extend indefinitely in all directions of the discrete plane. Special attention, however, will be given to the class of codes whose elements have finite supports and to the possibility of characterizing complete codes as the duals of the above class.

In the sequel, it will be convenient to represent the signals of $\mathscr{F}_{\infty}^{n}$ and, hence, the codewords of $\mathscr{E}$, via formal power series, by associating any sequence $\{w(i, j)\}$ with the series

$$
\sum_{i, j \in \mathbf{Z}} w(i, j) z_{1}^{i} z_{2}^{j}
$$

To avoid cumbersome notations, we will adopt the symbol w for denoting both the sequence and the associated power series (2.1). The context will always make clear which object we are referring to. Sometimes, mostly when a power series $\mathbf{v}$ is obtained as the (Cauchy) product of a series and a polynomial, it will be useful to denote the coefficient of $z_{1}^{i} z_{2}^{j}$ in $\mathrm{v}$ as $\left(\mathrm{v}, z_{1}^{i} z_{2}^{j}\right)$.

The main advantage in using formal power series is that many linear operators on $\mathscr{F}_{\infty}^{n}$ can be represented by appropriate matrices, with elements in $\mathscr{F}_{ \pm}:=$ $\mathrm{F}\left[z_{1}, z_{2}, z_{1}^{-1}, z_{2}^{-1}\right]$, the ring of 2-D Laurent polynomials ( $L-$ polynomials). This way, several fundamental operator properties find an immediate counterpart in terms of the structure of the corresponding matrices and, in particular; of their factors.

Definition: A matrix $G\left(z_{1}, z_{2}\right) \in \mathscr{F}_{ \pm}^{k \times n}$ is

- $\mathscr{F}_{ \pm}$-unimodular, if $k=n$ and $\operatorname{det} G$ is a unit in $\mathscr{F}_{ \pm}$;

- left factor prime $(\ell F P)$, if for every factorization $G=T \bar{G}$, with $T \in \mathscr{F}_{ \pm}^{k \times k}, T$ is $\mathscr{F}_{ \pm}$-unimodular;

- left zero prime $(\ell Z P)$, if the ideal generated by the maximal order minors of $G$ is the ring $\mathscr{F}_{ \pm}$itself.

Introducing a convolutional structure on $\mathscr{C}$ requires that we endow the set of its sequences with some closure properties, which constitute the mathematical formalization of very natural constraints of regularity. The most common requirements on $\mathscr{E}$ are linearity and shift invariance.

a) Linearity: If $\mathbf{w}_{1}$ and $w_{2}$ belong to $\mathscr{C}$, then $\alpha w_{1}+\beta w_{2}$ belongs to $\mathscr{C}$ for every $\alpha$ and $\beta$ in F.

b) Shift Invariance: $\mathbf{w} \in \mathscr{C}$ implies that $\mathbf{v}=z_{1}^{h} z_{2}^{k} \mathbf{w} \in \mathscr{C}$, $\forall h, k \in Z$, i.e. $\mathscr{C}$ is invariant with respect to the shifts in $\mathbf{Z} \times \mathbf{Z}$ along the coordinate axes.

As the set of formal power series $\mathscr{F}_{\infty}^{n}$ is naturally endowed with a module structure with respect to $\mathscr{F}_{ \pm}$, codes that satisfy properties a) and b) can be characterized as $\mathscr{F}_{ \pm}$-submodules of $\mathscr{F}_{\infty}^{n}$. They will be called admissible codes.

Example 1: Every submodule $\mathscr{C}$ of $\mathscr{F}_{ \pm}^{n}$ is an admissible code. Since $\mathscr{F}_{ \pm}^{n}$ is an $\mathscr{F}_{ \pm}$-Noetherian module [16], $\mathscr{C}$ is finitely generated, i.e., there exists a finite set of row vectors $\mathbf{g}_{1}, \mathbf{g}_{2}, \cdots, \mathbf{g}_{h}$ in $\mathscr{F}_{ \pm}^{n}$. such that

$$
\mathscr{E}=\left\{\sum_{i=1}^{h} a_{i} \mathbf{g}_{i}, a_{i} \in \mathscr{F}_{ \pm}\right\}=\left\{\mathbf{a} G, \mathbf{a} \in \mathscr{F}_{ \pm}^{h}\right\}=: \operatorname{Im}_{ \pm} G
$$

where $G$ denotes the polynomial matrix

$$
G=\operatorname{col}\left\{\mathbf{g}_{1}, \mathbf{g}_{2}, \cdots, \mathbf{g}_{h}\right\}
$$

Example 2: A sequence w has "past compact support" if, for every $(l, m) \in \mathbf{Z} \times \mathbf{Z}$, the corresponding past cone $\{(i, j): i \leq l, j \leq m\}$ intersects the support of $\mathbf{w}$ in a finite number of points.

The set

$$
\mathscr{E}=\left\{w \in \mathscr{F}_{\infty}^{n}: \operatorname{supp}(w) \text { past compact }\right\}
$$

is an admissible code. 
Example 3: Let $M_{1}, M_{2} \in \mathrm{F}^{\nu \times \nu}$ constitute a pair of commuting invertible matrices and let $K$ be in $\mathrm{F}^{\nu \times n}$. The set

$$
\mathscr{E}=\left\{\mathbf{w} \in \mathscr{F}_{\infty}^{n}: \mathbf{w}=\sum_{i, j \in \mathbf{Z}} x M_{1}^{i} M_{2}^{j} K z_{1}^{i} z_{2}^{j}, x \in \mathbf{F}^{\nu}\right\}
$$

is an admissible code. Moreover its dimension, as an F-vector space, is finite. It can be shown that all finitedimensional admissible codes have the above structure [17].

When testing whether a sequence $\mathbf{w}$ belongs to a code $\mathscr{C}$ that includes codewords with infinite support, the possibility of resorting to a finite set of autoregressive equations, applied at every point of $\mathbf{Z} \times \mathbf{Z}$, constitutes a very favorable situation. Actually, in this case, we can recognize a codeword by using only a finite set of samples $w(i, j)$ at each step of the testing procedure. Such a possibility clearly corresponds [7] to the existence of an $L$-polynomial matrix $H^{T}\left(z_{1}, z_{2}\right)$, such that

$$
\mathscr{C}=\operatorname{ker} H^{T}\left(z_{1}, z_{2}\right):=\left\{\mathbf{w} \in \mathscr{F}_{\infty}^{n}: \mathbf{w} H^{T}=\mathbf{0}\right\}
$$

and it can be restated as a closure property of the code, as follows.

c) Completeness: Let $\mathscr{S}_{1} \subset \mathscr{S}_{2} \subset \mathscr{S}_{3}, \cdots$, be a sequence of nested subsets such that $\bigcup_{m \in \mathbf{N}} \mathscr{S}_{m}=\mathbf{Z} \times \mathbf{Z}$ and let $w \in \mathscr{F}_{\infty}^{n}$. Then $w \in \mathscr{E}$ if (and only if) for every nonnegative integer $m$ there exists $\mathbf{v}_{m} \in \mathscr{E}$ such that

$$
\mathbf{v}_{m}\left|\mathscr{S}_{m}=\mathbf{w}\right| \mathscr{S}_{m}
$$

An equivalent way of stating the above property is the following. Introduce in $\mathbf{Z} \times \mathbf{Z}$ a distance function $d(\cdot, \cdot)$, by assuming

$$
\begin{aligned}
d[(i, j),(h, k)]=\mid i- & h|+| j-k \mid, \\
& \forall(i, j),(h, k) \in \mathbf{Z} \times \mathbf{Z}
\end{aligned}
$$

and define distance $\Delta(\cdot, \cdot)$ between two sequences $\mathbf{v}$ and $w$ in $\mathscr{F}_{\infty}^{n}$ as follows:

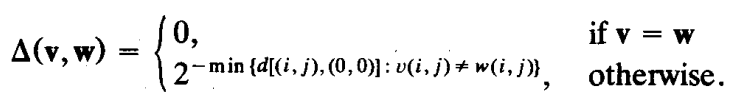

Then, $\mathscr{F}_{\infty}^{n}$ becomes a metric space, and property c) is exactly the completeness of $\mathscr{E}$ in the topology induced by the distance function $\Delta$. This means that if a sequence $\mathbf{v}_{1}, \mathbf{v}_{2}, \cdots$, in $\mathscr{C}$ converges to $\mathbf{w}$, then $\mathbf{w} \in \mathscr{E}$.

Proposition 2.1: Let $\mathscr{E} \subseteq \mathscr{F}_{\infty}^{n}$ be an admissible code. Then $\mathscr{C}$ is a complete code, i.e., it satisfies condition c) if and only if (2.2) holds.

Proof: The proposition above has been proved by $P$. Rocha in [7]. For an alternative proof see [18].

As an immediate corollary, we have that properties a)-c) are equivalent to the possibility of representing $\mathscr{C}$ as the kernel of an $L$-polynomial matrix.

Remark I: The codes considered in Examples 1 and 2 are not complete. On the other hand the code of Example
3 is complete, as it can be proved that it is the kernel of a polynomial matrix [18].

Given a finite window $\mathscr{S}$ and a set of samples, obtained by restricting to $\mathscr{S}$ a (possibly infinite) codeword $w$, it is interesting to investigate whether the data set, $w \mid \mathscr{S}$, can be completed into an appropriate finite codeword $\mathbf{v}$, whose support does not "exceed by too much" $\mathscr{S}$. If so, the values a codeword $w$ assumes on the window $\mathscr{S}$, constrain only the samples $w(i, j)$ in a finite neighborhood of it or, equivalently, do not provide any information on $w$ at points that are far enough from $\mathscr{S}$. Therefore, if no additional information on $w$ is available, we can always assume that the partial data at our disposal come from a finite codeword. The above property can be stated as follows.

d) Controllability: There exists a positive integer $\delta$ such that, for every finite set $\mathscr{S} \subset \mathbf{Z} \times \mathbf{Z}$ and every $\mathbf{v}_{1} \in \mathscr{E}$, there is a codeword $v_{2} \in \mathscr{C}$, such that (see Fig. 1)

$$
\mathbf{v}_{1}\left|\mathscr{S}=\mathbf{v}_{2}\right| \mathscr{S}
$$

and

$$
\operatorname{supp}\left(\mathbf{v}_{2}\right) \subseteq \mathscr{S}^{\delta}:=\{(i, j) \in \mathbf{Z} \times \mathbf{Z}: d((i, j), \mathscr{S})<\delta\} .
$$

A fundamental objective of coding theory is the investigation of the intrinsic structure of codes, without taking into account the way codewords are generated, and the analysis of those features with which good codes must be endowed. These should make them as efficient as possible with respect to design requirements, such as the distance among the codewords and the noise sensitivity.

Under the hypothesis that a $2-\mathrm{D}$ code $\mathscr{C}$ is complete, there are several equivalent formulations of the controllability property, which concern the internal structure of the codewords set. Some of them refer to the submodule of the finite codewords,

$$
\mathscr{C}_{f}:=\{w \in \mathscr{E}: \operatorname{supp}(\mathbf{w}) \text { finite }\}=\mathscr{E} \cap \mathscr{F}_{ \pm}^{n},
$$

others to the possibility of obtaining the code by a sort of "patching" of appropriate finite codewords.

$\left(d_{1}\right)$ : There exists a positive integer $\rho$ such that, given two disjoints subsets of $\mathbf{Z} \times \mathbf{Z}, \mathscr{S}_{1}$ and $\mathscr{S}_{2}$ whose distance $d$ is greater than $\rho$, and two codewords $w_{1}$ and $w_{2}$ in $\mathscr{C}$, there is a codeword $v \in \mathscr{E}$ such that

$$
\mathbf{w}_{1}\left|\mathscr{S}_{1}=\mathbf{v}\right| \mathscr{S}_{1} \text { and } \mathbf{w}_{2}\left|\mathscr{S}_{2}=\mathbf{v}\right| \mathscr{S}_{2} \text {. }
$$

This result can be rephrased as the possibility of "concatenating" two portions of distinct codewords into a single codeword, provided their supports are far enough apart [6]-[8].

$\left(d_{2}\right)$ : There exists a finite set $\mathscr{I}$ of finite support codewords with the property that $w \in \mathscr{F}_{\infty}^{n}$ belongs to $\mathscr{C}$ if and only if $w$ is represented as a locally finite sum of some, possibly shifted, elements of $\mathscr{I}$. So every codeword of $\mathscr{C}$ is obtained by resorting to an appropriate "covering" of the discrete plane with codewords and shifted codewords of $\mathscr{I}$.

$\left(d_{3}\right):$ The code $\mathscr{C}$ can be completely reconstructed from $\mathscr{C}_{f}$, the $\mathscr{F}_{ \pm}$-module of its finite codewords, by means of a 


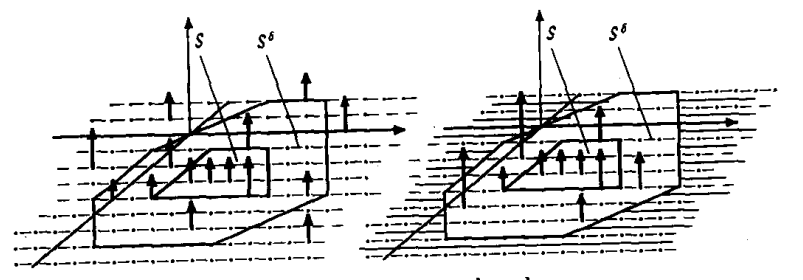

codeword $\mathbf{v}_{3}$

codeword $\mathrm{v}_{2}$

Fig. 1. Controllability of the code $\mathscr{E}$.

limit operation, namely, w belongs to $\mathscr{C}$ if and only if there is a sequence $\mathbf{w}_{1}, \mathbf{w}_{2}, \cdots$, in $\mathscr{C}_{f}$, converging to $\mathbf{w}$ in the sense of the pointwise topology. So $\mathscr{E}$ can be viewed as the closure of the module $\mathscr{C}_{f}$, that is, as the smallest (complete) code containing $\mathscr{C}_{f}$.

On the other hand, a code is naturally understood as the result of an encoding process applied to the information signals. Therefore, many concepts in coding theory are connected with the existence of an input-output transformation, whose image is the code itself. In this perspective, if the information signals are sequences in $\mathbf{Z} \times \mathbf{Z}$, with values in $\mathbf{F}^{k}$ for some integer $k$, and the code $\mathscr{E}$ is linear and shift invariant, it is natural to associate the transformation with a $k \times n \quad L$-polynomial matrix $G\left(z_{1}, z_{2}\right)$ and represent the code as

$$
\mathscr{C}=\operatorname{Im} G:=\left\{\mathbf{w}=\mathbf{u} G, \mathbf{u} \in \mathscr{F}_{\infty}^{k}\right\}
$$

As we shall see, property (2.5) is equivalent to the "internal" properties a)-d). Consequently, the convolutional nature of $\mathscr{E}$, i.e., the possibility of generating all codewords of $\mathscr{C}$ by convolving the input sequences with the matrix $G$ of the impulse response, has an exact counterpart in terms of the internal structure of the code, which can be characterized without any reference to the encoding process. We call convolutional a complete code satisfying condition d), or equivalently a code described as in (2.5).

Proposition 2.2, below, formalizes the main statements concerning the controllability property.

Proposition 2.2 (Equivalent Characterizations of Convolutional Codes): Let $\mathscr{E} \subseteq \mathscr{F}_{\infty}^{n}$. The following are equivalent:

1) $\mathscr{C}=\operatorname{Im} G, G \in \mathscr{F}_{ \pm}^{k \times n}$

2) $\mathscr{C}=\operatorname{ker} H^{T}, H^{T} \stackrel{ \pm}{\mathscr{F}^{n} \times p}, H^{T}$ right factor prime;

3d) $\mathscr{C}$ is complete and satisfies property $(d)$; $1,2,3$.

$\left.3 \mathrm{~d}_{\mathrm{i}}\right) \mathscr{C}$ is complete and satisfies property $\left(d_{i}\right), i=$

Proof: The equivalences $(1) \Leftrightarrow(2) \Leftrightarrow\left(3 d_{1}\right)$ have been proved by $P$. Rocha in [9]. An independent proof, based on the notion of duality, will be provided in Section IV. For the remaining equivalences we proceed by showing that $\left(3 d_{1}\right) \Leftrightarrow(3 d)$ and $\left(3 d_{3}\right) \Leftrightarrow(1) \Leftrightarrow\left(3 d_{2}\right)$.

$\left(3 d_{1}\right) \Rightarrow(3 d)$ : Take $\delta:=\rho$ and apply $\left(3 d_{1}\right)$ to $w_{1}:=v_{1}$, $\mathbf{w}_{2}=\mathbf{0}, \mathscr{S}_{1}:=\mathscr{S}$ (finite), and $\mathscr{S}_{2}:=C \mathscr{S}^{\delta}$, the complementary set of $\mathscr{S}^{\delta}$. The finite codeword $\mathbf{v}$ obtained in $\left(3 d_{1}\right)$ is the codeword $v_{2}$ we are looking for.

$(3 d) \Rightarrow\left(3 d_{1}\right)$ : The space $\mathscr{F}_{\infty}^{n}$ is sequentially compact, i.e., every sequence of elements in $\mathscr{F}_{\infty}^{n}$ contains a subse- quence which converges to an element in $\mathscr{F}_{\infty}{ }^{n}$. As a consequence of this property, one can show [18] that $(3 d)$ extends to the infinite subsets of $Z \times Z$. Set $\rho:=\delta$. There exist two codewords $\mathbf{v}_{1}$ and $\mathbf{v}_{2}$ such that $\mathbf{v}_{i}\left|\mathscr{S}_{i}=\mathbf{w}_{i}\right| \mathscr{S}_{i}$, $i=1,2$, and $\operatorname{supp}\left(\mathbf{v}_{i}\right) \subseteq \mathscr{S}_{i}^{\delta}$. The signal $\mathbf{v}:=\mathbf{v}_{1}+\mathbf{v}_{2}$ belongs to $\mathscr{E}$ and satisfies $\mathbf{v}\left|\mathscr{S}_{i}=\mathrm{v}_{i}\right| \mathscr{S}_{i}=\mathbf{w}_{i} \mid \mathscr{S}_{i}, i=1,2$, as required.

(1) $\Rightarrow\left(\mathbf{3 d}_{2}\right):$ Consider the set $\mathscr{I}:=\left\{\left[\alpha_{1} \alpha_{2}, \cdots\right.\right.$, $\left.\alpha_{k}\right] G, \alpha_{\nu} \in \mathrm{F}$, whose elements are the codewords corresponding to the "atomic" input signals $\left[\alpha_{1} \alpha_{2}, \cdots, \alpha_{k}\right] \in$ $\mathbf{F}^{k}$.

As $\mathbf{F}$ is a finite field, $\mathscr{I}$ is finite too. Every codeword in $\mathscr{B}$ can be written as

$$
\mathbf{w}=\mathbf{u} G=\sum_{i, j \in \mathbf{Z}} z_{1}^{i} z_{2}^{j}\left(\left[u_{1}(i, j) u_{2}(i, j) \cdots u_{k}(i, j)\right] G\right) .
$$

Since all codewords $\mathbf{w}_{i j}\left(z_{1}, z_{2}\right):=\left[u_{1}(i, j) u_{2}(i, j) \cdots\right.$ $\left.u_{k}(i, j)\right] G$ are elements of $\mathscr{F},(2.6)$ represents $w$ as a locally finite sum of elements and shifted elements of $\mathscr{F}$. $\left(3 \mathbf{d}_{2}\right) \Rightarrow(1)$ : Let $\mathscr{I}:=\left\{\mathbf{c}_{1}\left(z_{1}, z_{2}\right), \mathbf{c}_{2}\left(z_{1}, z_{2}\right)\right.$, $\left.\cdots, \mathbf{c}_{p}\left(z_{1}, z_{2}\right)\right\}$, with $\mathbf{c}_{i}\left(z_{1}, z_{2}\right) \in \mathscr{F}_{ \pm}^{n}, i=1,2, \cdots, p$. By assumption, the codewords in $\mathscr{C}$ are the elements in $\mathscr{F}_{\infty}^{n}$ which can be expressed as

$$
\begin{aligned}
& \sum_{i, j \in \mathbf{Z}} \sum_{t=1}^{p} \delta_{t}(i, j) z_{1}^{i} z_{2}^{j} \mathbf{c}_{t}\left(z_{1}, z_{2}\right) \\
& =\sum_{i, j \in \mathbf{Z}}\left[\delta_{1}(i, j) z_{1}^{i} z_{2}^{j} \quad \delta_{2}(i, j) z_{1}^{i} z_{2}^{j}\right. \\
& \left.\cdots \delta_{p}(i j) z_{1}^{i} z_{2}^{j}\right]\left[\begin{array}{c}
\mathbf{c}_{1}\left(z_{1}, z_{2}\right) \\
\mathbf{c}_{2}\left(z_{1}, z_{2}\right) \\
\ldots \\
\mathbf{c}_{p}\left(z_{1}, z_{2}\right)
\end{array}\right]
\end{aligned}
$$

where $\delta_{t}(\cdot, \cdot)$ takes values in $\{0,1\}$.

Letting $G\left(z_{1}, z_{2}\right)=\operatorname{col}\left(\mathbf{c}_{1}\left(z_{1}, z_{2}\right)\right.$, $\left.\mathbf{c}_{2}\left(z_{1}, z_{2}\right), \cdots, \mathbf{c}_{p}\left(z_{1}, z_{2}\right)\right\}$, we have that the $\mathscr{F}_{ \pm}$-module generated by the rows of $G$ is included in $\mathscr{C}$. We aim to prove that $\mathbf{u} G$ is in $\mathscr{C}$ for every $\mathbf{u} \in \mathscr{F}_{ \pm}^{p}$. Actually, given any sequence of finite nested sets $\mathscr{S}_{1} \subset \mathscr{S}_{2} \subset \cdots$ covering $\mathbf{Z} \times$ $\mathbf{Z}$, the sequence of input signals $\mathbf{u}_{\nu}$ defined by

$$
u_{\nu}(i, j)= \begin{cases}u(i, j) & \text { if }(i, j) \in \mathscr{S}_{\nu} \\ 0 & \text { otherwise. }\end{cases}
$$

converges to $\mathbf{u}$. By the continuity of $G[18], \mathbf{u}_{\nu} G$ converges to $\mathbf{u} G$. Since $\mathscr{C}$ is complete, $\mathbf{u}_{\nu} G \in \mathscr{C}, \forall \nu \Rightarrow \mathbf{u} G \in$ $\mathscr{C}$. Consequently, $\operatorname{Im} G \subseteq \mathscr{E}$.

On the other hand, by (2.7) every codeword can be expressed as the $G$-image of a series in $\mathscr{F}_{\infty}^{p}$ with coefficients in $\{0,1\}$. Therefore $\mathscr{C}=\operatorname{Im} G$.

(1) $\Rightarrow\left(3 d_{3}\right)$ : Let $\mathbf{w}$ be in $\mathscr{C}=\operatorname{Im} G$ and $w=u G$. Consider an $L$-polynomial sequence $\left\{\mathbf{u}_{\nu}\right\}$ converging to $\mathbf{u}$. Because of the continuity of $G$, the sequence of $L$-polynomial codewords $\left\{\mathbf{w}_{\nu}\right\}:=\left\{\mathbf{u}_{\nu} G\right\}$ converges to $\mathbf{w}=\mathbf{u} G$. 
$\left(3 d_{3}\right) \Rightarrow(1):$ The finite codewords of $\mathscr{C}$ constitute an $\mathscr{F}_{+}$-module $\mathscr{E}_{f}$, which is finitely generated as a submodule of $\mathscr{F}_{+}^{n}$. Let $\mathbf{g}_{\nu} \in \mathscr{F}_{+}^{n}, \nu=1,2, \cdots, k$, constitute a set of generators for $\mathscr{C}_{f}$ and $G:=\operatorname{col}\left\{\mathbf{g}_{1}, \mathbf{g}_{2}, \cdots, \mathbf{g}_{k}\right\}$. Clearly, $\operatorname{Im} G \subseteq \mathscr{C}$.

To prove the opposite inclusion, consider any codeword $\mathbf{w}$ in $\mathscr{C}$ and a sequence of finite codewords, $\mathbf{w}_{v} \in \mathscr{C}_{f}$, $\nu=1,2, \cdots$, converging to $w$. Since all finite codewords in $\mathscr{C}$ are linear combinations over $\mathscr{F}_{ \pm}$of the rows of $G$, there is a sequence $\left\{\mathbf{u}_{\nu}\right\}, \mathbf{u}_{\nu} \in \mathscr{F}_{+}^{k}$, such that $\left\{\mathbf{u}_{\nu} G\right\}=\left\{\mathbf{w}_{\nu}\right\}$. By the sequential compactness of $\mathscr{F}_{\infty}^{k}[18]$, we can extract from $\left\{\mathbf{u}_{v}\right\}$ a subsequence $\left\{\mathbf{u}_{\nu}\right\}$ that converges to some $\mathbf{u}$ in $\mathscr{F}_{\infty}^{k}$. So, by the continuity of the operator $G$, we have

$$
\mathbf{w}=\lim _{j \rightarrow \infty}\left(\mathbf{u}_{\nu_{j}} G\right)=\left(\lim _{j \rightarrow \infty} \mathbf{u}_{v_{j}}\right) G=\mathbf{u} G .
$$

It can be easily realized that, while a $k \times n$ polynomial matrix $G\left(z_{1}, z_{2}\right)$ uniquely identifies the convolutional code $\operatorname{Im} G=\left\{\mathbf{w}=\mathbf{u} G: \mathbf{u} \in \mathscr{F}_{\infty}^{k}\right\}$, the converse does not hold, as the same code $\mathscr{C}$ can be described as the image of different $L$-polynomial matrices. Two matrices $G_{1}\left(z_{1}, z_{2}\right)$ and $G_{2}\left(z_{1}, z_{2}\right)$, with elements in $\mathscr{F}_{ \pm}$and the same number of columns, are equivalent encoders if $\operatorname{Im} G_{1}=\operatorname{Im} G_{2}$. Since each convolutional code biuniquely corresponds to a class of equivalent encoders, the natural problems arise to investigate what conditions guarantee that two matrices belong to the same class and to find out in every equivalence class the most efficient encoders.

To answer these questions we need some preliminary results, concerning the relationships between the $\mathscr{F}_{ \pm^{-}}$ module

$$
\operatorname{Im}_{ \pm} G:=\left\{\mathbf{u} G: \mathbf{u} \in \mathscr{F}_{ \pm}^{k}\right\}
$$

and the $\mathscr{F}_{ \pm}$-submodule $\mathscr{E}_{f}$ of the finite codewords of $\mathscr{E}=\operatorname{Im} G$, that will be also denoted as $(\operatorname{Im} G)_{f}$.

Lemma 2.3 [17]: Let $\bar{G}\left(z_{1}, z_{2}\right)$ be a $k \times n \ell F P L$-polynomial matrix. Then

i) the $\mathscr{F}_{+}$-module $\operatorname{Im}_{+} \bar{G}$ is free;

ii) if $T\left(z_{1}, z_{2}\right)$ is a $k^{\prime} \times k L-$ polynomial matrix, of rank $k$ over $F\left(z_{1}, z_{2}\right)$, then $(\operatorname{Im} T \bar{G})_{f}$ coincides with $(\operatorname{Im} \bar{G})_{f}$

Lemma 2.4: Let $G\left(z_{1}, z_{2}\right)$ be a $k \times n L$-polynomial matrix, with full row rank over $\mathbf{F}\left(z_{1}, z_{2}\right)$. The following properties are equivalent.

i) $G\left(z_{1}, z_{2}\right)$ is $\ell F P$;

ii) the module $(\operatorname{Im} G)_{f}$ of the finite codewords in $\operatorname{Im} G$ coincides with $\operatorname{Im}_{+} G$, i.e., every finite codeword $\mathbf{w}$ of $\operatorname{Im} G$ is the image of a finite input sequence;

iii) $\operatorname{ker} G$, the $F$-vector space of all information sequences in $\mathscr{F}_{\infty}^{k}$ that produce the zero codeword, is finite dimensional.

Proof $i) \Rightarrow$ ii): Clearly $\operatorname{Im}_{ \pm} G$ is included in $(\operatorname{Im} G)_{f}$, since every linear combination in $\mathscr{F}_{ \pm}$of the rows of $G$ is a finite codeword of $\mathscr{C}$.

We aim to prove the opposite inclusion. The $\ell F P$ condition implies [5] the existence of two matrices $X\left(z_{1}, z_{2}\right)$ and $Y\left(z_{1}, z_{2}\right)$, with elements in $\mathscr{F}_{+}$, and two polynomials $h\left(z_{1}\right) \in \mathbf{F}\left[z_{1}, z_{1}^{-1}\right], k\left(z_{2}\right) \in \mathbf{F}\left[z_{2}, z_{2}^{-1}\right]$ such that (A2) holds. We therefore have

$$
\mathbf{w} X=\mathbf{u} G X=\mathbf{u} h\left(z_{1}\right), \quad \mathbf{w} Y=\mathbf{u} G Y=\mathbf{u} k\left(z_{2}\right)
$$

and, by multiplying the first equation by $k\left(z_{2}\right)$ and the second one by $h\left(z_{1}\right)$, we get $w X k\left(z_{2}\right)=w Y h\left(z_{1}\right)$. Since $h\left(z_{1}\right)$ and $k\left(z_{2}\right)$ are coprime, $h\left(z_{1}\right)$ is a common factor of all the components of $\mathbf{w} X$, that is there exists an $L$-polynomial vector $\mathbf{p}\left(z_{1}, z_{2}\right) \in \mathscr{F}_{ \pm}^{-k}$ such that $\mathbf{w} X=\mathbf{p} h\left(z_{1}\right)$. Thus, $h\left(z_{1}\right) \mathbf{w}=h\left(z_{1}\right)(\mathbf{u} G)=h\left(z_{1}\right)(\mathbf{p} G)$ and, consequently,

$$
h\left(z_{1}\right)(\mathbf{w}-\mathbf{p} G)=0 .
$$

Since all entries in (2.8) are in $\mathscr{F}_{ \pm}$, it follows that $\mathbf{w}=\mathbf{p} G$, and therefore $(\operatorname{Im} G)_{f}$ is included in $\operatorname{Im}_{+} G$.

ii) $\Rightarrow i$ ): Assume that $G$ is not $\ell F P$. As a consequence of Corollary A.2 in the Appendix, $G$ can be rewritten as $G=T \bar{G}$, where $\bar{G} \in \mathscr{F}_{ \pm}^{k \times n}$ is $\ell F P$ and $T \in$ $\mathscr{F}_{ \pm}^{k \times k}$ is a full rank nonunimodular matrix. Thus there exists a vector $\mathbf{p}\left(z_{1}, z_{2}\right) \in \mathscr{F}_{ \pm}^{k}$ such that equation

$$
\mathbf{u} T=\mathbf{p}
$$

has no solution in $\mathscr{F}_{ \pm}^{k}$. However, as $T$ is a full-rank square matrix, (2.9) admits a unique solution in $F\left(z_{1}, z_{2}\right)$ given by

$$
\mathbf{u}=\mathbf{p} T^{-1}=\mathbf{p} \frac{\operatorname{Adj} T}{\operatorname{det} T} .
$$

The entries of $\mathbf{u}$ can be viewed as series in $\mathscr{F}_{\infty}$, and hence $\mathbf{u}$ is an infinite input sequence in $\mathscr{F}_{\infty}^{k}$. We aim to show that $\mathbf{w}=\mathbf{u} G$ is a finite codeword that does not belong to $\operatorname{Im}_{ \pm} G$. Actually, $\mathbf{w}=\mathbf{u} G=(\mathbf{u} T) \bar{G}=\mathbf{p} \bar{G}$ is finite.

On the other hand, assume that there is a finite input sequence $\mathrm{v}$ such that $\mathrm{w}=\mathrm{v} G$. Then we have $\mathrm{w}=\mathrm{v} G=$ $(\mathbf{v} T) \bar{G}$, which implies $(\mathbf{p}-\mathbf{v} T) \bar{G}=0$. Since $\operatorname{Im}_{+} \bar{G}$ is a free module, we have $\mathbf{v} T=\mathbf{p}$, and (2.9) has an $L$-polynomial solution, a contradiction.

i) $\Leftrightarrow$ iii): $\operatorname{ker} G:=\left\{\mathbf{u} \in \mathscr{F}_{\infty}^{k}: \mathbf{u} G=0\right\}$ can be viewed as an autoregressive description of a complete behavior. It has been proved [9], [17] that a necessary and sufficient condition for a behavior being finite dimensional is that $G$ is $\ell F P$.

We are now in a position for introducing the basic results about the equivalence of two encoders.

Proposition 2.5 (Equivalent Encoders): Let $G_{1}\left(z_{1}, z_{2}\right)$ and $G_{2}\left(z_{1}, z_{2}\right)$ be two matrices with elements in $\mathscr{F}_{ \pm}$and dimensions $k_{1} \times n$ and $k_{2} \times n$, respectively. $G_{1}$ and $G_{2}$ are equivalent encoders if and only if

i) under the assumption that both $G_{1}\left(z_{1}, z_{2}\right)$ and $G_{2}\left(z_{1}, z_{2}\right)$ are $\ell F P$, we have

$$
\begin{aligned}
k_{1}=k_{2} \text { and } G_{2}\left(z_{1}, z_{2}\right)= & U\left(z_{1}, z_{2}\right) G_{1}\left(z_{1}, z_{2}\right), \\
& \text { with } U\left(z_{1}, z_{2}\right) \text { unimodular }
\end{aligned}
$$

ii) under the assumption that $G_{1}\left(z_{1}, z_{2}\right)$ is $\ell F P$, there is a $k_{2} \times k_{1}$ full-column-rank $L$-polynomial matrix, $P_{1}\left(z_{1}, z_{2}\right)$, such that $G_{2}=P_{1} G_{1}$;

iii) in the general case, there exist two full column rank $L$-polynomial matrices $P_{1}\left(z_{1}, z_{2}\right)$ and $P_{2}\left(z_{1}, z_{2}\right)$, of suitable dimensions, such that

$$
P_{1} G_{1}=P_{2} G_{2} \text {. }
$$


Proof: Given a full-column-rank matrix $P \in \mathscr{F}_{ \pm}^{h \times k}$, the map $P: \mathscr{F}_{\infty}^{h} \rightarrow \mathscr{F}_{\infty}^{k}: \mathbf{u} \mapsto \mathbf{u} P$ is onto. Consequently the convolutional codes $\operatorname{Im} G$ and $\operatorname{Im} P G$ coincide for any $G \in \mathscr{F}_{ \pm}^{k \times n}$. Thus (2.10) in iii), and in particular $G_{2}=U G_{1}$ and $G_{2}=P_{1} G_{1}$ in i) and ii), imply that $G_{1}$ and $G_{2}$ are equivalent encoders.

Conversely, assume first that $G_{1}$ and $G_{2}$ are $\ell F P$ equivalent encoders. Then, by property $\left(d_{3}\right)$ and Lemma 2.4 we have

$$
\operatorname{Im}_{ \pm} G_{1}=\left(\operatorname{Im} G_{1}\right)_{f}=\left(\operatorname{Im} G_{2}\right)_{f}=\operatorname{Im}_{ \pm} G_{2} .
$$

As each row of $G_{1}$ (of $G_{2}$ ) is an $\mathscr{F}_{ \pm}$-linear combination of the rows of $G_{2}$ (of $G_{1}$ ), there exist $L$-polynomial matrices $P_{1}$ and $P_{2}$ such that $P_{1} G_{1}=G_{2}$ and $P_{2} G_{2}=G_{1}$. We have then $G_{1}=P_{1} P_{2} G_{1}$ and $G_{2}=P_{2} P_{1} G_{2}$. The $\ell F P$ property yields

$$
P_{1} P_{2}=I_{k_{1}}, \quad P_{2} P_{1}=I_{k_{2}}
$$

showing that $k_{1}=k_{2}$ and both $P_{1}$ and $P_{2}$ are unimodular.

Assume next that $G_{1}$ and $G_{2}$ are equivalent encoders, and only $G_{1}$ is $\ell F P$. By Corollary A.2, $G_{2}$ can be factorized as $G_{2}=T \bar{G}_{2}$, where $\bar{G}_{2}$ is $\ell F P$ and $T$ full-column rank. Thus, $\operatorname{Im} G_{2}=\operatorname{Im} \bar{G}_{2}$ and, consequently, $G_{1}$ and $\bar{G}_{2}$ are $\ell F P$ equivalent encoders. It follows that $\bar{G}_{2}=U G_{1}$, for a suitable unimodular matrix $U$, and, letting $P_{1}=T U$, one gets $G_{2}=P_{1} G_{1}$, as required.

Finally, case iii), suppose that $G_{1}$ and $G_{2}$ are equivalent encoders, and neither $G_{1}$ nor $G_{2}$ are $\ell F P$. Clearly, we have $G_{i}=\tilde{T}_{i} \bar{G}_{i}, i=1,2$, with $\tilde{T}_{i}$ full-column-rank and $\bar{G}_{i}$ $\ell F P$ matrices. Moreover, $\bar{G}_{1}=U \bar{G}_{2}$ for some $\mathscr{F}_{ \pm}$-unimodular matrix $U$. So, letting $T_{1}=T_{1} U$ and $T_{2}=T_{2}$ we get

$$
G_{1}=T_{1} \bar{G}_{2}, \quad G_{2}=T_{2} \bar{G}_{2}
$$

Consider any pair of $L$-polynomial matrices $X_{1}$ and $X_{2}$ with the property that $A_{i}:=\left[\begin{array}{ll}T_{i} & X_{i}\end{array}\right], i=1,2$, is a nonsingular $k_{i} \times k_{i} L$-polynomial matrix, and assume $k_{1} \geq k_{2}$. Then we have

$$
A_{1}^{-1} G_{1}=\left[\begin{array}{c}
\bar{G}_{2} \\
0 \\
0
\end{array}\right]=\left[\begin{array}{c}
A_{2}^{-1} \\
0
\end{array}\right]
$$

Introduce the following left matrix fraction description (MFD)

$$
\left[\begin{array}{c}
A_{2}^{-1} \\
0
\end{array}\right]=L^{-1} N
$$

with $N$ full-column rank, and rewrite $A_{1} L^{-1}$ as a left MFD, $A_{1} L^{-1}=Q^{-1}$ B. As $G_{1}=A_{1} L^{-1} N G_{2}=$ $Q^{-1} B N G_{2}$, we end up with $Q G_{1}=B N G_{2}$, which corresponds to (2.10), upon assuming $P_{1}=Q$ and $P_{2}=B N$.

Remark II: It is worthwhile to underline that every convolutional code $\mathscr{E}$ can be represented as the image of a $\ell F P$ matrix. Actually, given any encoder $G\left(z_{1}, z_{2}\right)$ of $\mathscr{C}$, by Corollary A.2 we can extract a greatest left factor, obtaining

$$
G\left(z_{1}, z_{2}\right)=T\left(z_{1}, z_{2}\right) \bar{G}\left(z_{1}, z_{2}\right)
$$

with $T$ full-column rank and $\bar{G} \ell F P$. By the above proposition, $\bar{G}\left(z_{1}, z_{2}\right)$ is a $\ell F P$ encoder of $\mathscr{C}$.

\section{INJECTIVITY AND DeCODING}

The purpose of an encoding scheme is to associate every input sequence with a specific codeword that preserves the information message, but is less sensitive to the action of noise. So, in order to make possible the retrieval of the original message at the decoding stage, it is quite obvious that every codeword has to be the image of a unique information sequence, which amounts to assuming that the map from the input space $\mathscr{F}_{\infty}^{k}$ to the codewords space $\mathscr{C}$ is injective.

As proved in the previous section, a convolutional code can be expressed as the image or the kernel of appropriate Laurent polynomial matrices. The following proposition shows that the injectivity requirements reduces to a zero primeness condition on the above matrices. This entails some relevant consequences on both the internal properties of the code $\mathscr{E}$ and the classes of encoders and decoders of $\mathscr{E}$.

Proposition 3.1 (Injective Encoders): Let $\mathscr{E}$ be a convolutional code of length $n$ and rank $k$. The following are equivalent:

i) $\mathscr{E}$ admits an injective encoder;

ii) $\mathscr{E}=\operatorname{Im} G\left(z_{1}, z_{2}\right), G \in \mathscr{F}_{ \pm}^{k \times n} \ell Z P$;

iii) $\mathscr{C}=\operatorname{ker} H^{T}\left(z_{1}, z_{2}\right), H^{T} \in \mathscr{F}_{ \pm}^{n \times(n-k)} r Z P$.

Proof $i) \Leftrightarrow i i$ ): If $G$ is $\ell \mathrm{ZP}$, by Proposition A.3 there exists an $n \times k$ matrix, $K\left(z_{1}, z_{2}\right)$, with elements in $\mathscr{F}_{ \pm}$, such that $G K=I_{k}$. So, $\mathbf{u} G=0$ implies $0=(\mathbf{u} G) K=$ $\mathbf{u}(G K)=\mathbf{u}$, which means that $G$ defines an injective input-output map.

Conversely, we aim to prove that, if $G$ is not $\ell \mathrm{ZP}$, it is not an injective encoder. If rank $G<k$, the result is trivial, so we confine ourselves to the case rank $G=k$.

Consider first the case when $G$ is not $\ell$ FP. By Corollary A.2, there exist two $L$-polynomial matrices, $\bar{G}\left(z_{1}, z_{2}\right)$, $k \times n$ and $\ell \mathrm{FP}$, and $T\left(z_{1}, z_{2}\right), k \times k$ with $\operatorname{det} T \neq 0$ and not a unit in $\mathscr{F}_{ \pm}$, such that $G=T \bar{G}$.

- If det $T$ is not a unit in $\mathbf{F}\left(z_{1}\right)\left[z_{2}, z_{2}^{-1}\right]$, in the (renormalized) Hermite form [2], [5] of $T$ with respect to $\mathbf{F}\left[z_{1}, z_{1}^{-1}\right]\left[z_{2}, z_{2}^{-1}\right]$, we have $S\left(z_{1}, z_{2}\right)=L\left(z_{1}, z_{2}\right) T\left(z_{1}, z_{2}\right)$, where $S \in \mathscr{F}_{ \pm}^{k \times k}$ is upper triangular, and $L \in \mathscr{F}_{ \pm}^{k \times k}$ has determinant in $\mathbf{F}\left[z_{1}, z_{1}^{-1}\right]$.

As $\operatorname{det} S=\operatorname{det} T \operatorname{det} L$, the assumption $o n \operatorname{det} T \mathrm{im}$ plies that at least one diagonal element in $S$ is a nonunit polynomial in $\mathrm{F}\left(z_{1}\right)\left[z_{2}, z_{2}^{-1}\right]$. Let $S_{i i}\left(z_{1}, z_{2}\right)$ be the first element with this property, and consider $v_{i}\left(z_{1}, z_{2}\right)$, a series in $\mathscr{F}_{\infty}$ such that

$$
v_{i} S_{i i}=0 \quad \text { and } \quad v_{i} \operatorname{det} L \neq 0 .
$$

Then there exists a vector $\mathrm{v} \in \mathscr{F}_{\infty}^{k}$, with the first $i-1$ entries identically zero, such that $v S=0$. On the other hand $\mathbf{v} L$ is not zero, otherwise $0=\mathrm{v} L \operatorname{Adj} L=\mathrm{v} \operatorname{det} L$ would imply $v_{i} \operatorname{det} L=0$, which contradicts (3.1). So 
$\mathrm{v} L\left(z_{1}, z_{2}\right)$ is a nonzero element in $\operatorname{ker} T$, and hence, in $\operatorname{ker} G$.

- If det $T$ is a unit in $\mathbf{F}\left(z_{1}\right)\left[z_{2}, z_{2}^{-1}\right]$, it cannot be also a unit in $\mathbf{F}\left(z_{2}\right)\left[z_{1}, z_{1}^{-1}\right]$, otherwise $\operatorname{det} T$ would be a unit in $F_{ \pm}$. So we can resort to the Hermite form of $T$ with respect to $\left[z_{2}, z_{2}^{-1}\right]\left[z_{1}, z_{1}^{-1}\right]$ to prove that $\operatorname{ker} G$ is nontrivial.

When $G$ is $\ell$ FP (but not $\ell \mathrm{ZP}$ ), ker $G$ is a finite dimensional vector space [14], and $G$ is not an injective encoder.

ii) $\Leftrightarrow$ iii): By Proposition 2.2 and Remark II, the equivalence of ii) and iii) holds for factor prime matrices. Since for every $\mathbf{u} \in \mathscr{F}_{\infty}^{k},(\mathbf{u} G) H^{T}=\mathbf{u}\left(G H^{T}\right)=0$, it follows that $G H^{T}=0$, and therefore, by Proposition A.4, $G$ is $\ell \mathrm{ZP}$ if and only if $H^{T}$ is $r \mathrm{ZP}$.

Given a convolutional code $\mathscr{C}=\operatorname{Im} G$, of length $n$ and rank $k$, it is natural to wonder whether it admits injective encoders. Clearly injective (i.e., $\ell \mathrm{ZP}$ ) encoders, if any, have to be looked for among the $\ell$ FP ones. On the other hand, by Proposition 2.5 , if $\bar{G}\left(z_{1}, z_{2}\right)$ is a $k \times n \ell$ FP encoder of $\mathscr{C}$, any other $\ell$ FP encoder is given by $\tilde{G}=U \bar{G}$, $U$ unimodular. Since the premultiplication by $\mathscr{F}_{ \pm}$-unimodular matrices preserves the $\ell$ ZP property, the existence of a $\ell \mathrm{ZP}$ encoder implies that all $\ell \mathrm{FP}$ encoders are $\ell$ ZP. Unlike the 1-D case, left factor-primeness does not imply left zero-primeness, and examples can be given of 2-D convolutional codes devoid of injective encoders.

Example 3: Let $\mathbf{F}=G F(2)$. It is easy to check that the following $L$-polynomial matrix

$$
G_{1}\left(z_{1}, z_{2}\right)=\left[\begin{array}{ccc}
z_{1}^{-1}+1 & 0 & z_{1}^{2} \\
z_{2}^{-1} & z_{2}+1 & 0
\end{array}\right]
$$

is $\ell \mathrm{ZP}$, as the only input sequence producing the zero codeword is $\mathbf{u}=\mathbf{0}$.

On the contrary,

$$
G_{2}\left(z_{1}, z_{2}\right)=\left[\begin{array}{ccc}
z_{1}^{2}+1 & 0 & z_{1} \\
z_{2}+1 & z_{2}^{2}+z_{1} & 0
\end{array}\right]
$$

is $\ell$ FP but not $\ell \mathrm{ZP}$, since all maximal order minors have a common zero in $(1,1)$. Therefore, $G_{2}$ is not an injective encoder. It can be easily realized that $\mathbf{u}=\left[\begin{array}{ll}0 & \sum_{i, j} z_{1}^{i} z_{2}^{j}\end{array}\right]$ is the unique nonzero input sequence in $\operatorname{ker} G_{2}$.

According to the above discussion, we can single out among 2-D convolutional codes those that admit a $\ell$ ZP encoder. They will be called basic, in analogy with the 1-D case [1], and will be characterized by the possibility of extending somehow finite sequences into codewords.

A complete code $\mathscr{C}$, and, a fortiori, a convolutional one, is a submodule of $\mathscr{F}_{\infty}^{n}$ whose elements satisfy a finite set of autoregressive equations, the parity checks of the code. By associating each equation with an $L$-polynomial column vector $\mathbf{h}_{i}^{T}\left(z_{1}, z_{2}\right)$, we have that $\mathbf{w} \in \mathscr{C}$ if and only if $\mathbf{w h}_{i}^{T}=0, i=1,2, \cdots, p$. So, by juxtaposing the columns $\mathbf{h}_{i}^{T}$ into a matrix $H^{T}=\operatorname{col}\left\{\mathbf{h}_{1}^{T}, \mathbf{h}_{2}^{T}, \cdots, \mathbf{h}_{p}^{T}\right\}$, we get the usual kernel representation $\mathscr{C}=\operatorname{ker} H^{T}=\left\{\mathrm{w} \in \mathscr{F}_{\infty}^{n}: \mathrm{w} H^{T}=0\right\}$.

Definition: A sequence $\mathrm{v} \in \mathscr{F}_{\infty}^{n}$ satisfies the parity checks of the code in $(r, s) \in \mathbf{Z} \times \mathbf{Z}$ if

$$
\left(\mathbf{v} H^{T}, z_{1}^{\mu} z_{2}^{\nu}\right)=0, \quad \forall(\mu, \nu) \in(r, s)+\operatorname{supp}\left(H^{T}\right),
$$

where $(r, s)+\operatorname{supp}\left(H^{T}\right):=\{(r+i, s+j):(i, j) \in$ $\operatorname{supp}\left(H^{T}\right)$ \}.

More generally, if $\mathscr{T}$ is an arbitrary subset of $\mathbf{Z} \times \mathbf{Z}$, $\mathbf{v}$ satisfies the parity checks of the code on $\mathscr{T}$, if it satisfies them in every point $(r, s) \in \mathscr{T}$, that is

$$
\left(\mathrm{v} H^{T}, z_{1}^{\mu} z_{2}^{\nu}\right)=0, \quad \forall(\mu, \nu) \in \mathscr{T}+\operatorname{supp}\left(H^{T}\right)
$$

where $\mathscr{T}+\operatorname{supp}\left(H^{T}\right):=\bigcup_{(r, s) \in \mathscr{T}}\left\{(r, s)+\operatorname{supp}\left(H^{T}\right)\right\}$

Letting $H^{T}\left(z_{1}, z_{2}\right)=\sum_{i j} H_{i j}^{T} z_{1}^{i} z_{2}^{j}$, condition (3.2) reduces to the following system of linear equations:

$$
\begin{aligned}
& \sum_{(i, j) \in \operatorname{supp}\left(H^{T}\right)} v(\mu-i, \nu-j) H_{i j}^{T}=0, \\
& \forall(\mu, \nu) \in(r, s)+\operatorname{supp}\left(H^{T}\right),
\end{aligned}
$$

and hence to the system of all difference equations which involve the sample $v(r, s)$. Analogously, $v$ meets condition (3.3) if all difference equations involving the samples $v(r, s)$, with $(r, s)$ in $\mathscr{T}$, are satisfied. In Fig. 2, each dashed polygon intersecting $\mathscr{T}$ represents the coordinates $(\mu-$ $i, \nu-j$ ) of the samples which appear in a system like (3.4).

Clearly, when verifying whether $\mathbf{v}$ satisfies the parity checks of the code on $\mathscr{F}$, we take into account not only the samples on $\mathscr{T}$, but also those which belong to an appropriate set $\tilde{\mathscr{T}} \supseteq \mathscr{T}$. The remaining tests we have to perform, when deciding whether $\mathbf{v}$ is a codeword, are represented by systems of difference equations that involve only the samples of $\mathbf{v}$ on $C \mathscr{T}$. Some of them, however, utilize again the samples on $\tilde{\mathscr{T}} \backslash \mathscr{F}$, as suggested by the checkered polygon in Fig. 2. So, it could happen that the data on $\mathscr{\mathscr { T }}$ satisfy the parity checks on $\mathscr{T}$, yet no selection of the data on $C \tilde{\mathscr{T}}$ makes possible the fulfillment of the parity checks on $C \tilde{\mathscr{F}}$. Otherwise stated, the specific assignment of the values of $\mathbf{v}$ on $\tilde{\mathscr{T}}$ compromises any possibility of extending the data on $\tilde{\mathscr{T}}$ into a legal codeword. In these situations, the natural question arises whether such an extension could be made possible by changing only the data which are "close" to the border of $\tilde{\mathscr{T}}$. More precisely, we wonder whether there is a positive integer $\delta$, such that any sequence $\mathbf{v}$, satisfying the parity checks of the code on $\mathscr{T}^{\delta}$, can be modified into a codeword $\mathbf{w}$, which coincides with $\mathbf{v}$ on the window $\mathscr{T}$. A positive answer is very important from the syndrome decoder point of view. Actually, when the parity checks of the code are verified in $\mathscr{S}^{\delta}$, we can assume the restriction $\mathbf{v} \mid \mathscr{T}$ as correct and, whenever the parity checks fail in some point $(r, s) \notin \mathscr{T}^{\delta}$, we have to modify only the values of $\mathbf{v}$ on $C 9$.

Generally, neither the completeness assumption nor the more restrictive hypothesis that $\mathscr{E}$ is a convolutional code, imply that the code $\mathscr{E}$ exhibits the aforementioned features. As we shall see, these constitute the exact coun- 


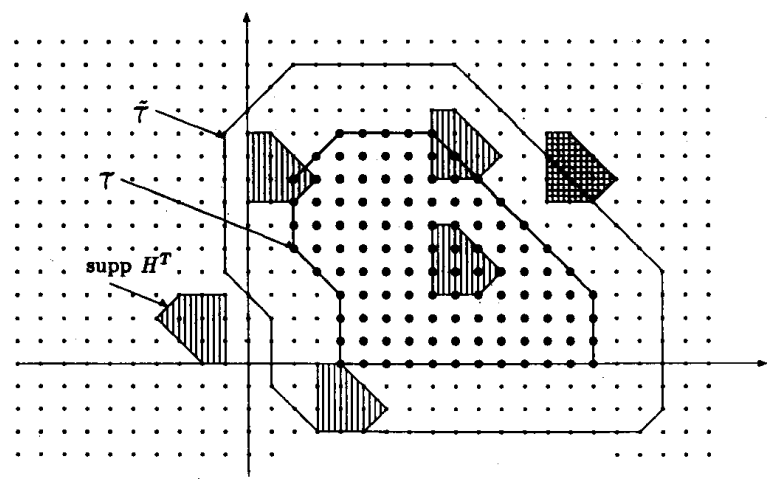

Fig. 2. Parity checks locations.

terpart, from an internal point of view, of the condition for the existence of an injective encoder (stated in Proposition 3.1), and provide an equivalent definition of 2-D basic codes. The formal definition of these properties will be assumed as a further constraint on the structure of $\mathscr{E}$.

e) Extendability: Let $\mathscr{C}=\operatorname{ker} H^{T}$. There exists a positive integer $\boldsymbol{\delta}$ such that, for every finite subset $\mathscr{S} \subset \mathbf{Z} \times \mathbf{Z}$ and every $\mathrm{v} \in \mathscr{F}_{\infty}^{n}$, which satisfies on $\mathscr{S}^{\delta}$ the parity checks of the code, there exists a codeword $w \in \mathscr{C}$ such that

$$
\mathbf{w}|\mathscr{S}=\mathbf{v}| \mathscr{S} .
$$

Lemma 3.2: Let $\mathscr{C}=\operatorname{ker} H^{T}\left(z_{1}, z_{2}\right)$ be a code satisfying the extendability property. Then property e) holds for all (not necessarily finite) subsets of $\mathbf{Z} \times \mathbf{Z}$.

Proof: Assume that the sequence $\mathrm{v} \in \mathscr{F}_{\infty}^{n}$ satisfies the parity checks of the code on an infinite set $\mathscr{S}^{\delta}$, and let $\mathscr{S}_{1} \subset \mathscr{S}_{2} \subset \mathscr{S}_{3} \subset, \cdots$, be a sequence of finite nested sets in $\mathbf{Z} \times \mathbf{Z}$ such that $\bigcup_{m} \mathscr{S}_{m}=\mathscr{S}$. Since $\mathbf{v}$ satisfies the parity checks on $\mathscr{S}_{i}^{\delta}, i=1,2,3, \cdots$, there exists a sequence of codewords $\mathbf{w}_{i}, i=1,2,3, \cdots$, such that $\mathbf{w}_{i}\left|\mathscr{S}_{i}=\mathbf{v}\right| \mathscr{S}_{i}$. As $\mathscr{F}_{\infty}^{n}$ is sequentially compact, there is a subsequence $\left\{\mathbf{w}_{\nu}\right\}$ of $\left\{w_{i}\right\}$ converging to $w \in \mathscr{F}_{\infty}^{n}$. The proposition is proved by observing that

- as $\mathbf{w}_{\boldsymbol{v}} \in \mathscr{C}$ for every $j$, by the completeness of $\mathscr{C}$, $\mathbf{w} \in \mathscr{C}$

- since $\mathbf{w}\left|\mathscr{S}_{\nu_{j}}=\mathbf{w}_{\nu_{j}}\right| \mathscr{S}_{\nu_{j}}=\mathbf{v} \mid \mathscr{S}_{v_{j}}$ for every $\nu_{j}$, it follows that $\mathbf{w}|\mathscr{S}=\mathbf{v}| \mathscr{S}$.

Proposition 3.3: Extendability Implies Controllability

Proof: Let $w \in \mathscr{C}$, with $\mathscr{C}$ a code satisfying property e), and consider a finite set $\mathscr{S}$. Define $\mathrm{v} \in \mathscr{F}_{\infty}^{n}$ as follows:

$$
v(h, k)= \begin{cases}0, & \text { for every }(h, k) \in \mathscr{S}^{\delta} \\ w(h, k), & \text { otherwise }\end{cases}
$$

where $\delta$ is like in e).

Since v satisfies the parity checks in $\mathscr{T}=\mathscr{S} \cup C\left(\mathscr{S}^{2 \delta}\right)$, by the previous lemma there is a codeword $\overline{\mathbf{v}} \in \mathscr{E}$ such that $\bar{v}|\mathscr{G}=\mathbf{v}| \mathscr{T}$. Clearly $(\mathbf{w}-\bar{v})$ is in $\mathscr{C}$, and $(\mathbf{w}-\bar{v}) \mid \mathscr{S}=$ $\mathbf{w} \mid \mathscr{S}$. Moreover, $(w-\bar{v}) \mid C\left(\mathscr{S}^{2 \delta}\right)=0$, implies that supp (w $-\bar{v}) \subseteq \mathscr{S}^{2 \delta}$, so $\mathscr{C}$ satisfies property d).

Extendability and controllability are very close to each other. To further highlight the strict connection between the two notions, we will also show that, for a complete code, property e) is equivalent to the following property $e_{1}$ ), (which represents the natural counterpart of $\left(d_{1}\right)$, and hence is called "strong controllability" in [9]).

$\left.e_{1}\right)$ Let $\mathscr{C}=k e r H^{T}$. There exists a positive integer $\rho$ such that for every pair of subsets $\mathscr{S}_{1}$ and $\mathscr{S}_{2}$ of $Z \times Z$, with $d\left(\mathscr{S}_{1}, \mathscr{S}_{2}\right)>2 \rho$, and for every pair of sequences $v_{1}, v_{2} \in \mathscr{F}_{\infty}^{n}$, which satisfy the parity checks of the code on $\mathscr{S}_{1}^{\rho}$ and $\mathscr{S}_{2}^{\rho}$ respectively, a codeword $w \in \mathscr{B}$ exists, such that

$$
\mathbf{w}\left|\mathscr{S}_{1}=\mathbf{v}\right| \mathscr{S}_{1} \text { and } \mathbf{w}\left|\mathscr{S}_{2}=\mathbf{v}\right| \mathscr{S}_{2} \text {. }
$$

The difference between properties d) and e) might at first appear somewhat elusive, because of the mathematically involved character of their definitions. It can be best understood, however, when one looks for a coding interpretation of both properties. As a consequence of the proof of Proposition 3.3, e) implies that, given any sequence $\mathbf{v} \in \mathscr{F}_{ \pm}^{n}$ that satisfies the parity checks of the code in $\mathscr{S}^{\delta}$, there is a codeword $\mathbf{w}$ that coincides with $\mathbf{v}$ in $\mathscr{S}$ and whose support does not exceed $\mathscr{S}^{2 \delta}$. Note that no a priori information is needed, guaranteeing that the data on the window $\mathscr{S}$ are the restriction of a legal codeword.

This information is needed, on the contrary, in the case of simple controllability, when trying to extend the available data into a finite codeword. As in practice one expects that only a finite set of data be available and, anyhow, only a finite number of parity checks could be performed on them, in most cases the above information is beyond our reach.

The proof of the equivalence between e) and $e_{1}$ ) is included in the following summarizing proposition, which provides a complete picture of the connections between basic codes, introduced from an "external" point of view in Proposition 3.1, and codes endowed with the extendability property.

Proposition 3.4 (Equivalent Characterizations of Basic Codes): Let $\mathscr{C} \subseteq \mathscr{F}_{\infty}^{n}$. The following are equivalent:

1) $\mathscr{E}=\operatorname{Im} G, G \in \mathscr{F}_{+}^{k \times n} / \mathrm{ZP}$

2) $\mathscr{C}=\operatorname{ker} H^{T}, H^{T} \stackrel{ \pm}{\in} \mathscr{F}_{ \pm}^{n \times(n-k)} r \mathrm{ZP}$;

3e) $\mathscr{C}$ satisfies property e);

$\left.3 \mathrm{e}_{1}\right) \mathscr{C}$ satisfies property $\mathrm{e}_{1}$ ).

Proof $(1) \Leftrightarrow$ (2): See Proposition 3.1.

(2) $\Rightarrow(3 e)$ : Assume that $H^{T} \in \mathscr{F}_{ \pm}^{n \times(n-k)}$ is $r \mathrm{ZP}$ and $W \in \mathscr{F}_{ \pm}^{(n-k) \times n}$ is a polynomial left inverse of $H^{T}$.

Let $\delta_{1}=\max \left\{|i|+|j|:\left(H^{T}, z_{1}^{i} z_{2}^{j}\right) \neq 0\right\}, \quad \delta_{2}=\max \{|i|$ $\left.+|j|:\left(W, z_{1}^{i} z_{2}^{j}\right) \neq 0\right\}$, and $\delta=\delta_{1}+\delta_{2}$. If $v \in \mathscr{F}_{\infty}^{n}$ satisfies the parity checks of the code on a finite set $\mathscr{S}^{\delta} \subseteq \mathbf{Z} \times \mathbf{Z}$, the series $\mathbf{a}=\mathbf{v} H^{T}$ satisfies $\left(a, z_{1}^{\mu}, z_{2}^{\nu}\right)=0$ for every $(\mu, \nu) \in \mathscr{S}^{\delta}+\operatorname{supp}\left(H^{T}\right)$. Since $\operatorname{supp}\left(H^{T}\right) \subseteq \mathscr{S}^{\delta_{1}}$, we have

$$
\mathscr{S}^{\delta_{2}} \subseteq \mathscr{S}^{\delta}+\operatorname{supp}\left(H^{T}\right) \subseteq C[\operatorname{supp}(\mathbf{a})] .
$$

Introduce next the series $\mathbf{x}=\mathbf{a} W$. As $\operatorname{supp}(\mathbf{x}) \subseteq \operatorname{supp}(\mathbf{a})$ $+\operatorname{supp}(W)$ and $\operatorname{supp}(\mathbf{a}) \subseteq C \mathscr{S}^{\delta_{2}}$, it follows that

$$
\operatorname{supp}(\mathbf{x}) \subseteq C \mathscr{S}^{\delta_{2}}+\operatorname{supp}(W) \subseteq\left(C \mathscr{S}^{\delta_{2}}\right)^{\delta_{2}}=C \mathscr{S}
$$

and therefore $\mathbf{x} \mid \mathscr{S}=\mathbf{0}$. 
Finally, let $w:=\mathbf{v}-\mathbf{x}$. As a consequence of $\mathbf{x} H^{T}=$ $\mathbf{a} W H^{T}=\mathbf{a}=\mathbf{v} H^{T}$, we have $\mathbf{w} H^{T}=(\mathbf{v}-\mathbf{x}) H^{T}=0$, which implies $\mathbf{w} \in \mathscr{C}$. Moreover $\mathbf{w}|\mathscr{S}=(\mathbf{v}-\mathbf{x})| \mathscr{S}=\mathbf{v} \mid \mathscr{S}$.

$(3 e) \Rightarrow(2)$ : Let $\mathscr{C}$ satisfy the extension property. Then $\mathscr{E}$ is a convolutional code, and can be described as $\mathscr{C}=$ $\operatorname{ker} H^{T}$, with $H^{T} r \mathrm{FP}$. To prove that $H^{T}$ is $r \mathrm{ZP}$, by Proposition A5 it is sufficient to show that the equation

$$
\mathbf{x} H^{T}=\mathbf{a}
$$

admits an $L$-polynomial solution for all vectors a in $\mathscr{F}_{ \pm}^{n-k}$.

As $H^{T}$ has full-column rank over $\mathbf{F}\left(z_{1}, z_{2}\right),(3.7)$ admits a solution $\mathrm{v} \in \mathscr{F}_{\infty}^{n}$. To complete the proof we will show that there is a codeword $\mathbf{w}$, differing from $\mathrm{v}$ on a finite set $\mathscr{T}$. Actually, in this case we have

$$
(\mathbf{v}-\mathbf{w}) H^{T}=\mathbf{a}, \quad \operatorname{supp}(\mathbf{v}-\mathbf{w}) \subseteq \mathscr{T}
$$

and, consequently, $(\mathbf{v}-\mathbf{w})$ is an $L$-polynomial solution of (3.7). set

By assumption, v satisfies the parity checks of $\mathscr{E}$ on the

$$
\mathscr{H}=\left\{(r, s):\left((r, s)+\operatorname{supp}\left(H^{T}\right)\right) \cap \operatorname{supp}(\mathbf{a})=\varnothing\right\}
$$

whose complement is a finite set

Clearly, (see Fig. 3), a set $\mathscr{S}$ exists such that $C \mathscr{S}$ is finite and $\mathscr{S}^{\delta} \subseteq \mathscr{H}$. As v satisfies the parity checks of the code in $\mathscr{S}^{\delta}$, by $3 \mathrm{e}$ ) there is a codeword $w$ such that $\mathbf{w}|\mathscr{S}=\mathbf{v}| \mathscr{S}$. Therefore $\mathbf{v}$ and $\mathbf{w}$ differ only on a (finite) subset of $C \mathscr{P}$.

$(3 e) \Leftrightarrow\left(3 e_{1}\right):$ The proof can be performed along the same lines followed in showing the equivalence (3d) $\Leftrightarrow$ $\left(3 d_{1}\right)$ in Proposition 2.2.

Once an encoder converts an information message into the corresponding codeword, the encoded message $w$ is transmitted over a noisy channel. Generally, the received sequence $\mathbf{r}$ not only differs from the original message $\mathbf{w}$, but also does not belong to the code $\mathscr{C}$. So we need to project $\mathbf{r}$ on the space of codewords, in order to find out the best approximation to $\mathbf{r}$ in $\mathscr{E}$, namely a codeword $\hat{\mathbf{r}}$ whose distance from $\mathbf{r}$ is minimal. Often $\hat{\mathbf{r}}$ differs from the transmitted codeword $w$ by a nonzero reconstruction error

$$
\mathbf{e}=\hat{\mathbf{r}}-\mathbf{w}
$$

which is a codeword too. Having no possibility of finding e, all we can do is to reconstruct $\hat{\mathbf{u}}$, the input sequence corresponding to $\hat{\mathbf{r}}$, and assume it as an approximation of the correct information sequence. This step is performed by a decoder, namely a right inverse of the encoder matrix $G\left(z_{1}, z_{2}\right)$, which produces the sequence $\hat{\mathbf{u}}$, when receiving $\hat{\mathbf{r}}=\hat{\mathbf{u}} G$ as its input.

It can be easily realized from (3.8) that if there exist finite codewords generated by infinite information sequences, then a finite error $e$ in the reconstruction of the codeword $\mathbf{w}$ could produce an infinite error when decoding $\hat{\mathbf{r}}$ instead of $\mathbf{w}$. Such catastrophic errors, however, are avoided when, to preserve the injectivity property, we confine ourselves to the class of basic encoders and, therefore, to codes that satisfy the extendability property.

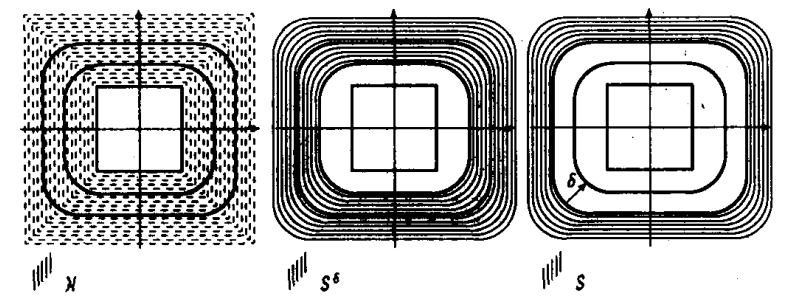

Fig. 3. Set inclusions in Proposition 3.4.

Indeed, if $G$ is a left zero prime $L$-polynomial matrix, it admits at least one $L$-polynomial right inverse $G^{-1}\left(z_{1}, z_{2}\right)$, and therefore every finite codeword $e$ in $\mathscr{C}$ is generated by one (and only one) finite input sequence $u_{e}=e G^{-1}$. An interesting consequence of the above reasoning is that, when achieving the injectivity of a convolutional encoder, one also guarantees the existence of a polynomial decoder, thus ruling out the possibility of catastrophic errors.

Remark $I V$ : As $G$ defines an injective input-output map, a decoder represented by a rational right inverse $G^{-1}\left(z_{1}, z_{2}\right)$ of $G$ would associate to any finite codeword e in $\mathscr{E}$, the same input sequence $\mathbf{u}_{e}$ as the polynomial decoder. In this case, however, expression $\mathbf{e} G^{-1}$ would be meaningless when $e$ is not polynomial, and we should restrict our attention to codewords whose supports do not extend to the whole discrete plane.

\section{Dual Codes and Syndrome Decoders}

The structure of 2-D codes, as discussed in Section II, can be clarified further through the duality relation between finite and complete codes. When referring to a finite code $\mathscr{C}$ of length $n$, we mean (see Example 1 of Section II) a submodule of $\mathscr{F}_{ \pm}^{n}$ defined as $\mathscr{E}=\operatorname{Im}_{ \pm} G=$ $\left\{\mathbf{w}=\mathbf{u} G: \mathbf{u} \in \mathscr{F}_{ \pm}^{h}\right\}$, where $G$ denotes an arbitrary matrix in $\mathscr{F}_{ \pm}^{h \times n}$. On the other hand, a complete (and, in particular, a convolutional) code $\mathscr{D}$ of length $n$ is defined as $\mathscr{D}=\operatorname{ker} H^{T}=\left\{\mathrm{w} \in \mathscr{F}_{\infty}^{n}: \mathrm{w} H^{T}=0\right\}$, with $H^{T}$ an arbitrary matrix in $\mathscr{F}_{ \pm}^{n \times q}$. The two are dual concepts that interact in the encoding and decoding processes. In most cases, it is quite reasonable to assume that 2-D information signals are finite support, and, therefore, finite codes are easily regarded as the result of an encoding operation. Even if complete and convolutional codes can be introduced by simply extending this point of view to infinite information signals, it is very convenient to give them a different interpretation in algebraic terms.

A complete code is more naturally viewed as a family of F-valued linear functions on the space of finite sequences, via the canonical algebraic duality [17] between $\mathscr{F}_{ \pm}^{n}$ and the space of linear functionals $L\left(\mathscr{F}_{ \pm}^{n}\right)$. So our philosophy will be to characterize a finite code $\mathscr{C}$ as the set of codewords that are in the kernel of a suitable space of linear functionals, and conversely, a (complete) dual code $\mathscr{D}$ as the set of linear parity checks necessary to decide whether a finite sequence is a legal codeword. 
The duality properties find an obvious application in the synthesis of syndrome decoders. Indeed, a complete characterization of the syndrome decoders of $\mathscr{C}$ can be achieved by resorting to a systematic analysis of the class of its dual codes.

Introduce in $\mathscr{F}_{ \pm}^{m} \times \mathscr{F}_{\infty}^{m}$ the following nondegenerate bilinear form:

$$
\langle\cdot, \cdot\rangle_{m}: \mathscr{F}_{ \pm}^{m} \times \mathscr{F}_{\infty}^{m} \rightarrow F
$$

defined by $\langle\mathbf{u}, \mathbf{v}\rangle_{m}=\left(\mathbf{u v}^{T}, 1\right)=\sum_{i, j \in \mathbf{z}} u(i, j) v^{T}(-i,-j)$. Two vectors $\mathrm{u} \in \mathscr{F}_{ \pm}^{m}$ and $\mathrm{v} \in \mathscr{F}_{\infty}^{m}$ are called orthogonal if $\langle\mathbf{u}, \mathbf{v}\rangle_{m}=0$. Given any submodule $\mathscr{H}^{m}$ of $\mathscr{F}_{+}^{m}$, its orthogonal complement $\mathscr{M}^{\perp}$, comprises all the vectors of $\mathscr{F}_{\infty}^{m}$ which are orthogonal to $\mathscr{K}$. Similarly, every submodule $\mathscr{N}$ of $\mathscr{F}_{\infty}^{m}$ identifies an orthogonal complement $\mathscr{N}^{\perp}$ in $\mathscr{F}_{ \pm}^{m}$.

The space $\mathscr{F}_{\infty}^{m}$ can be viewed as $L\left(\mathscr{F}_{ \pm}^{m}\right)$, the algebraic dual of $\mathscr{F}_{ \pm}^{m}$. In fact, we can associate with every $v \in \mathscr{F}_{\infty}^{m}$ the linear functional on $\mathscr{F}_{ \pm}^{m}$ defined by

$$
f_{v}(\cdot)=\langle\cdot, \mathbf{v}\rangle_{m}
$$

and, conversely, every linear functional on $\mathscr{F}_{ \pm}^{m}$ can be represented as in (4.1) for an appropriate choice of $\mathbf{v} \in$ $\mathscr{F}_{\infty}^{m}$. The identification of $\mathscr{F}_{\infty}^{m}$ with $L\left(\mathscr{F}_{ \pm}^{m}\right)$ makes it possible to use some results, not valid for arbitrary pairs of dual spaces [19].

Let $\mathscr{C}$ be a finite code, described as the image of the map

$$
G: \mathscr{F}_{ \pm}^{k} \rightarrow \mathscr{F}_{ \pm}^{n}: \mathbf{u} \mapsto \mathbf{u} G
$$

and consider the map

$$
G^{T}: \mathscr{F}_{\infty}^{n} \rightarrow \mathscr{F}_{\infty}^{k}: \mathrm{v} \mapsto \mathrm{v} G^{T} .
$$

The mappings $G$ and $G^{T}$ are dual, since $\langle\mathbf{u} G, \mathbf{v}\rangle_{n}=$ $\left(\mathbf{u} G \mathbf{v}^{T}, 1\right)=\left(\mathbf{u}\left(\mathbf{v} G^{T}\right)^{T}, 1\right)=\left\langle\mathbf{u}, \mathbf{v} G^{T}\right\rangle_{k}$. By resorting to the well-known relations

$$
\text { (A) }\left(\operatorname{Im}_{ \pm} G\right)^{\perp}=\operatorname{ker} G^{T}\left(\operatorname{ker} G^{T}\right)^{\perp}=\operatorname{Im}_{ \pm} G
$$

we induce a bijective correspondence between finite codes of length $n$, represented as images of appropriate $L$-polynomial matrices, and complete codes of the same length, described as kernels of $L$-polynomial matrices. This correspondence associates a finite code $\operatorname{Im}_{+} G$, with its dual, namely the $\mathscr{F}_{ \pm}$-module $\operatorname{ker} G^{T} \subseteq \mathscr{F}_{\infty}^{n}$ of all the parity checks of the code. Conversely, the dual of a complete code $\operatorname{ker} G^{T}$ is the module $\operatorname{Im}_{ \pm} G \subseteq \mathscr{F}_{ \pm}^{k}$ of its parity checks.

As a straightforward consequence of $(\mathbf{A})$, one gets

$$
\text { (B) }\left(\operatorname{Im}_{ \pm} G\right)^{\perp \perp}=\operatorname{Im}_{ \pm} G \quad\left(\operatorname{ker} G^{T}\right)^{\perp \perp}=\operatorname{ker} G^{T}
$$

which means that every code can be exactly reconstructed from the space of its parity checks.

The duality between complete and finite codes can be better understood by analyzing the correspondence between convolutional codes and a particular subclass of finite codes. As we have seen, every complete code can be described as the kernel, in $\mathscr{F}_{\infty}^{n}$, of an $L$-polynomial matrix, while only a convolutional code, i.e., the kernel of a rFP matrix, can be represented as the image of an $L$-polynomial matrix (see [9] and Proposition 2.2). Lemma 4.1, below, shows that for finite codes a dual situation holds. Actually they are always the images of $L$-polynomial matrices, but only the images of $\ell$ FP matrices can be expressed as kernels.

Lemma 4.1: Let $\mathscr{E}$ be a submodule of $\mathscr{F}_{ \pm}^{n}$. Then $\mathscr{C}$ is the kernel of an $L$-polynomial matrix if and only if there exists an $/$ FP matrix, $\bar{G}\left(z_{1}, z_{2}\right)$, such that $\mathscr{G}=\operatorname{Im}_{ \pm} \bar{G}$.

Proof: Let $\mathscr{G}=\operatorname{Im}_{ \pm} \bar{G}$ with $\bar{G} \in \mathscr{F}_{ \pm}^{k \times n} \ell \mathrm{FP}$, and consider a full-column rank matrix, $H^{T} \in \mathscr{F}_{ \pm}^{n \times(n-k)}$, such that $\bar{G} H^{T}=0$. Clearly, if $\mathbf{w} \in \mathscr{C}$, then $\mathbf{w}=\mathbf{u} \bar{G}$ for some $\mathbf{u} \in \mathscr{F}_{ \pm}^{k}$, and $\mathbf{w} H^{T}=(\mathbf{u} \bar{G}) H^{T}=\mathbf{u}\left(G H^{T}\right)=0$, so $\mathbf{w} \in$ $\operatorname{Ker}_{ \pm} \stackrel{H}{ }^{T}$. On the other hand, if $w \in \mathscr{F}_{ \pm}^{n}$ is in $\operatorname{ker}_{ \pm} H^{T}$, it belongs to the subspace of $F\left(z_{1}, z_{2}\right)^{n}$ orthogonal to the columns of $H^{T}$, and spanned by the rows of $\bar{G}$. Then there exists $f \in F\left(z_{1}, z_{2}\right)^{k}$ such that $f \bar{G}=\mathbf{w}$. As $\bar{G}$ is $\ell$ FP, by Lemma A.1 $\mathrm{f}$ can be chosen in $\mathscr{F}_{ \pm}^{k}$. So $w$ belongs to $\operatorname{Im}_{ \pm} \vec{G}$.

Conversely, let $\mathscr{C}=\mathrm{ker}_{+} H^{T}$ and consider any $\ell \mathrm{FP}$ matrix $\bar{G}\left(z_{1}, z_{2}\right) \in \mathscr{F}_{ \pm}^{(n-p) \times p}$ such that $\bar{G} \bar{H}^{T}=0$. Using the same arguments as in the first part of the proof, one shows that $\mathscr{E}=\operatorname{Im}_{ \pm} \bar{G}$.

By completing the duality relations (A) with

(C) $\left(\operatorname{Im} G^{T}\right)^{\perp}=\operatorname{ker}_{ \pm} G\left(\operatorname{ker}_{ \pm} G\right)^{\perp}=\operatorname{Im} G^{T}$ one immediately proves the proposition below.

Proposition 4.2: Let $G: \mathscr{F}_{ \pm}^{k} \rightarrow \mathscr{F}_{ \pm}^{n}$ and $G^{T}: \mathscr{F}_{\infty}^{n} \rightarrow \mathscr{F}_{\infty}^{k}$ be dual mappings. The following are equivalent:

1) the finite code $\operatorname{Im}_{ \pm} G$ can be represented as the kernel of an $L$-polynomial matrix;

2) the complete code $\operatorname{ker} G^{T}$ is convolutional, i.e., it can be described as the image of an $L$-polynomial matrix.

Remark V: Properties (A) and (C) together with Lemma 4.1 allow us to obtain an alternative proof of the equivalence between 1) and 2) in Proposition 2.2.

Actually, if $\mathscr{D}=\operatorname{Im} G^{T}$ is a convolutional code, as a consequence of (C) $\mathscr{D}$ is the dual of the finite code $\mathscr{C}:=\operatorname{ker}_{ \pm} G$. Then, by Lemma 4.1 , there exists a $/$ FP matrix $H\left(z_{1}, z_{2}\right)$ such that $\mathscr{C}=\operatorname{Im}_{ \pm} H$ and hence, by (A), $\mathscr{D}=\left(\operatorname{Im}_{ \pm} H\right)^{\perp}=\operatorname{ker} H^{T}$. So $\mathscr{D}$ is the kernel of a $r$ FP $L$-polynomial matrix. Conversely, if $\mathscr{D}=\operatorname{ker} H^{T}$ is a complete code and $H^{T}\left(z_{1}, z_{2}\right)$ is $r F P$, by (A) $\mathscr{D}$ is the dual of the finite code $\mathscr{C}:=\operatorname{Im}_{ \pm} H$, with $H \ell$ FP. By Lemma 4.1, there exists a matrix $G\left(z_{1}, z_{2}\right)$ such that $\mathscr{C}=\operatorname{ker}_{ \pm} G$, and therefore, by $(C), \mathscr{D}=\operatorname{Im} G^{T}$ is a convolutional code.

It is quite clear that every code described as the kernel of an $L$-polynomial matrix $H^{T}\left(z_{1}, z_{2}\right)$ admits $H^{T}$ as a syndrome decoder, since a sequence $\mathbf{v}$ belongs to the code if and only if $\mathbf{v} H^{T}=0$. Hence, every complete code admits a syndrome decoder, while, among finite codes, only those that are the image of an $/ F P$ matrix have this property. When a (finite or infinite) code, assigned through the encoder $G$, can be represented as the kernel of an $L$-polynomial matrix, the following proposition provides an algorithm to find a syndrome decoder. 
Proposition 4.3: (i) If $\mathscr{E}=\operatorname{Im}_{ \pm} \bar{G}$ is a finite code with $\bar{G}\left(z_{1}, z_{2}\right) \in \mathscr{F}_{ \pm}^{k \times n} \ell \mathrm{FP}$, every $L$-polynomial matrix $H^{T}\left(z_{1}, z_{2}\right)$ of rank $n-k$, satisfying $\bar{G} H^{T}=0$, is a syndrome decoder of $\mathscr{B}$;

(ii) If $\mathscr{E}=\operatorname{Im} G$ is a convolutional code of rank $k$, then every $r$ FP $L$-polynomial matrix $\bar{H}^{T}\left(z_{1}, z_{2}\right)$ of rank $n-k$ satisfying $G \vec{H}^{T}=0$ is a syndrome decoder of $\mathscr{C}$.

Proof: (i) It is obvious that, if $w$ belongs to $\mathscr{E}$, then $\mathbf{w}=\mathbf{u} \bar{G}, \mathbf{u} \in \mathscr{F}_{ \pm}^{k}$, satisfies $\mathbf{w} H^{T}=\mathbf{u} \bar{G} H^{T}=0$. Conversely, every $w \in \mathscr{F}^{n}$ satisfying $w H^{T}=0$, belongs to the subspace of $\mathbf{F}\left(z_{1}, z_{2}\right)^{n}$ orthogonal to the columns of $H^{T}\left(z_{1}, z_{2}\right)$, which is spanned by the rows of $\bar{G}$. As $\bar{G}$ is $\ell$ FP, by Lemma $A .1 w$ is a linear combination over $\mathscr{F}_{ \pm}$of the rows of $\bar{G}$, and therefore $w \in \mathscr{E}$.

(ii) After factorizing $G$ into $G=L \bar{G}$, with $\bar{G} \ell \mathrm{FP}$, the convolutional code $\mathscr{E}$ can be equivalently represented as $\mathscr{C}=\operatorname{Im} \bar{G}$ and condition $G \bar{H}^{T}=0$ is equivalent to $\bar{G} \bar{H}^{T}=0$. So we need only to prove

$$
\operatorname{Im} \bar{G}=\operatorname{ker} \bar{H}^{T} .
$$

Since $\bar{H}^{T}$ is $r \mathrm{FP}, \operatorname{ker} \bar{H}^{T}$ is convolutional. As convolutional codes can be uniquely reconstructed from the submodule of the finite codewords [see $\left(d_{3}\right)$ ], it will be sufficient to show that (4.2) holds when restricted to $\mathscr{F}_{ \pm}^{n}$, namely $(\operatorname{Im} \bar{G})_{f}=\operatorname{Im}_{ \pm} \bar{G}=\operatorname{ker}_{ \pm} \bar{H}^{T}$. But that is just what has been shown in part (i).

Note that the number of the parity checks we have to apply to a sequence $\mathrm{v}$ does not exceed the number of the columns of $H^{T}\left(z_{1}, z_{2}\right)$. Implementing a parity check, however, generally involves an infinite number of steps, unless $\mathscr{C}$ is a finite code, described as ker ${ }_{ \pm} H^{T}$. In this case only a finite number of steps is required to decide whether $\mathbf{v}$ belongs to $\mathscr{E}$, if an upper bound on the diameter of its support is a priori known.

We conclude this section by focusing our attention on the problem of obtaining syndrome decoders for a finite code $\mathscr{C}$ that cannot be represented as the kernel of an $L$-polynomial matrix. As a general result, we already know that, if $\mathscr{B}=\operatorname{Im}_{ \pm} G$ is a finite code, the dual code $\mathscr{D}=$ ker $G^{T}$ allows us to identify $\mathscr{C}$ as $\mathscr{D}^{\perp}$. The differences from case i) in Proposition 4.3 come from the fact that a representation of $\mathscr{D}$ as the image of an $L$-polynomial matrix is no longer available. As the module of the parity checks cannot be generated by the columns of an $L$-polynomial matrix, the best we can do is to extract from

$$
\mathscr{D}=\operatorname{ker} G^{T}=\left\{\mathrm{v} \in \mathscr{F}_{\infty}^{n}: \mathrm{v} G^{T}=0\right\}
$$

the submodule

$$
\mathscr{D}_{f}:=\operatorname{ker}_{ \pm} G^{T}=\left\{\mathrm{v} \in \mathscr{F}_{ \pm}^{n}: \mathbf{v} G^{T}=0\right\}
$$

and to represent it as the module generated by the rows of an $n \times p /$ FP matrix $\bar{H}\left(z_{1}, z_{2}\right)$, i.e., $\mathscr{D}_{f}=\operatorname{Im}_{ \pm} \bar{H}$. Clearly, any codeword $\mathrm{w} \in \mathscr{C}$ satisfies $\mathrm{w} \bar{H}^{T}=0$. Letting $G=L \bar{G}$, with $\bar{G} \ell \mathrm{FP}$, we have that the $\mathscr{F}_{+}$-module of the finite sequences in $\operatorname{ker} \bar{H}^{T}$ is given by $\overline{\mathscr{C}}^{ \pm}=\operatorname{Im}_{ \pm} \bar{G} \supsetneqq \mathscr{C}$ where the inclusion is proper because of the assumption on $\mathscr{E}$. This means that the syndrome decoder $\bar{H}^{T}$ accepts as legal codewords even sequences in $\overline{\mathscr{E}} \backslash \mathscr{E}$ that are not elements of the code.

\section{State-Space Realization of ENCODERS AND DECODERS}

Assigning an encoder via an $L$-polynomial matrix $G\left(z_{1}, z_{2}\right)$ corresponds to describing the algorithm that transforms an input information sequence into an output codeword, and hence to specifying only what happens at the terminals of an encoding device. The realization problem consists of obtaining a mathematical model of some "machine" that implements the input-output map. In other words, a state-space realization shows how the encoding algorithm proceeds, by explicitly displaying the corresponding evolution of the memory function.

In general there is not a unique way to find an algorithm that produces the input-output map of a convolutional encoder. So we have to introduce some a priori assumptions, as in the 1-D case, on the class of the mathematical models to use for this purpose. Moreover, as there is no natural notion of causality in the discrete plane, we need also to specify the partial ordering which underlies the recursive data processing. The class of 2-D models more extensively investigated in the literature is that of 2-D systems, for which the state equation updates according to a quarter plane causality notion. In this section we shall analyze to what extent 2 -D systems can be used for realizing 2-D encoders and decoders.

A (first-order quarter-plane causal) 2-D system $\Sigma=$ ( $A_{1}, A_{2}, B_{1}, B_{2}, C, D$ ) is given by the following equations [14]:

$$
\begin{gathered}
x(i+1, j+1)=x(i, j+1) A_{1}+x(i+1, j) A_{2} \\
+u(i, j+1) B_{1}+u(i+1, j) B_{2} \\
w(i, j)=x(i, j) C+u(i, j) D
\end{gathered}
$$

where the local state $x(i, j)$ is a $\nu$-dimensional vector over $\mathbf{F}$, input and output functions take values in $\mathbf{F}^{k}$ and $\mathbf{F}^{n}$ respectively, and $A_{1}, A_{2}, B_{1}, B_{2}, C$, and $D$ are matrices of suitable dimensions, with entries in $F$. When trying to eliminate the state variables of a 2-D system, in order to make explicit the input-output relation it produces, some restrictive hypotheses have to be introduced on both the supports of the input signals and the initial conditions of the system, which are formalized as follows.

(i) (Past Finite Support of the Input $u$ ): For every $(l, m)$ $\in \mathbf{Z} \times \mathbf{Z}$, the corresponding past cone $\{(i, j): i \leq l, j \leq m\}$ intersects the support of $\mathbf{u}$ in a finite number of points.

(ii) (Zero Initial Conditions): For every $(l, m) \in \mathbf{Z} \times \mathbf{Z}$, $\operatorname{supp}(\mathbf{u}) \cap\{(i, j): i \leq l, j \leq m\}=\varnothing$ implies $x(l, m)=0$.

Under assumptions (i) and (ii), the system output $w$ corresponding to the input sequence $\mathbf{u}$ is given by $\mathbf{w}=$ $\mathbf{u} G_{\Sigma}$, where

$$
G_{\Sigma}\left(z_{1}, z_{2}\right)=D+\left(B_{1} z_{1}+B_{2} z_{2}\right)\left(I-A_{1} z_{1}-A_{2} z_{2}\right)^{-1} C
$$

is the transfer matrix of $\Sigma$. 
It is a well-known result [20] that every proper rational 2-D matrix $G\left(z_{1}, z_{2}\right)$ is the transfer matrix of a suitable 2-D-state model $\Sigma$. In other words, when considering an input-output map $\gamma: \mathbf{u} \mapsto \mathbf{w}=\mathbf{u} G$ on the space of sequences with past compact support, there exist 2-D-state models (5.1) that produce $\mathbf{u} G$ as an output, when feeded with the input $\mathbf{u}$.

Since a convolutional (in particular, basic) code $\mathscr{E}$ can always be thought of as the image of a $k \times n$ matrix $G$ with elements in $\mathrm{F}\left[z_{1}, z_{2}\right]$, the submodule of the codewords of $\mathscr{E}$ with past finite support can be generated by an appropriate-state model $\Sigma$ as given in (5.1), whose transfer matrix $G_{\Sigma}$ coincides with $G$. Indeed, when condition (ii) is met, such codewords of $\mathscr{E}$ are obtained by applying to $\Sigma$ all input sequences with past finite supports, and the correspondence between inputs and outputs of $\Sigma$ is exactly the map between information sequences and codewords, generated by the encoder $G$.

Clearly, $\Sigma$ can be synthesized by resorting to suitable connections of adders, multipliers, and shift registers in both directions of the discrete plane, and therefore will be called a "realization" (or a state model) of the encoder $G$.

Given an encoder $G$, there are infinitely many models (5.1) that realize $G$, i.e., infinitely many sextuples of matrices $\Sigma=\left(A_{1}, A_{2}, B_{1}, B_{2}, C, D\right)$ that satisfy the equation

$$
G\left(z_{1}, z_{2}\right)=D+\left(B_{1} z_{1}+B_{2} z_{2}\right)\left(I-A_{1} z_{1}-A_{2} z_{2}\right)^{-1} C .
$$

These realizations, however, are by no means equivalent to each other. In particular, the local state-spaces of different realizations of $G$ need not have the same dimension, and realizations with minimal dimension are not necessarily related by a change of basis in the local-state space. So, the problem arises of characterizing in some way what realizations are the most reliable ones. To that purpose, various additional aspects have to be taken into account.

a) If the state-output transfer matrix ( $I-A_{1} z_{1}-$ $\left.A_{2} z_{2}\right)^{-1} C$ is not polynomial, local states $x$ exist, which give rise to free output evolutions $x\left(I-A_{1} z_{1}-A_{2} x_{2}\right)^{-1} C$ with infinite supports. Clearly, such local states, when induced by noise, generate infinite error sequences in the encoding process.

b) If the input-state transfer matrix $\left(B_{1} z_{1}+B_{2} z_{2}\right)(I-$ $\left.A_{1} z_{1}-A_{2} z_{2}\right)^{-1}$ is not polynomial, finite support input sequences possibly produce infinite support sequences in the state space. Therefore, $\Sigma$ could remain indefinitely excited by a finite signal, even through the corresponding output dies out in a finite number of steps.

Both the previous drawbacks can be avoided if the inverse matrix $\left(I-A_{1} z_{1}-A_{2} z_{2}\right)^{-1}$ is polynomial or, equivalently [13], if the characteristic polynomial of the system is unitary, that is

$$
\operatorname{det}\left(I-A_{1} z_{1}-A_{2} z_{2}\right)=1 \text {. }
$$

2-D systems satisfying condition (5.3) are called "finite memory," since they reach the zero state in a finite number of steps after zeroing the input signal. Since every matrix $G\left(z_{1}, z_{2}\right) \in \mathbf{F}\left[z_{1}, z_{2}\right]^{k \times n}$ admits a finite memory realization [19], it follows that every polynomial encoder, and a fortiori every basic polynomial encoder, can be synthesized by resorting to a 2-D system with finite memory.

c) When implementing a complete transmission system, we have to realize both the encoder and the corresponding decoder via finite memory 2-D state models (5.1), and hence to use an encoder-decoder pair with elements in $\mathrm{F}\left[z_{1}, z_{2}\right]$. So, the code $\mathscr{C}$ has to be the image of a matrix $G$ that is $\ell \mathrm{ZP}$ not only over $\mathscr{F}_{ \pm}$(which amounts to assuming that $\mathscr{C}$ is basic) but also over $\mathrm{F}\left[z_{1}, z_{2}\right]$. Namely, the ideal generated in $\mathrm{F}\left[z_{1}, z_{2}\right]$ by the maximal order minors of $G$ is the ring $F\left[z_{1}, z_{2}\right]$ itself. This condition guarantees the existence of a right inverse $G^{-1}$ with elements in $\mathrm{F}\left[z_{1}, z_{2}\right]$, so that both $G$ and $G^{-1}$ have finite memory realizations.

The following proposition characterizes $2-\mathrm{D}$ basic codes that admit an encoder, $\ell \mathrm{ZP}$ in $\mathbf{F}\left[z_{1}, z_{2}\right]$.

Proposition 5.1 [Basic Encoders in $F\left(z_{1}, z_{2}\right)$ ]: Let $\mathscr{C}$ be a basic code of length $n$ and rank $k$. The following are equivalent:

(i) there exists a basic encoder $G_{+}\left(z_{1}, z_{2}\right) \in$ F $\left[z_{1}, z_{2}\right]^{k \times n}$ that is $\ell \mathrm{ZP}$ in $\mathbf{F}\left[z_{1}, z_{2}\right]$;

(ii) for any basic encoder $G\left(z_{1}, z_{2}\right) \in \mathscr{F}_{ \pm}^{k \times n}$, with maximal order minors $m_{i}(G), i=1,2, \cdots, N=\left(\begin{array}{l}n \\ k\end{array}\right)$, there is a pair $(l, m) \in \mathbf{Z} \times \mathbf{Z}$ such that

$$
\bigcup_{i=1}^{N} \operatorname{supp}\left(m_{i}(G) z_{1}^{l} z_{2}^{m}\right) \subseteq \mathbf{N} \times \mathbf{N}
$$

and the ideal in $\mathrm{F}\left[z_{1}, z_{2}\right]$ generated by $m_{i}(G) z_{1}^{l} z_{2}^{m}, i=$ $1,2, \cdots, N$ is the whole ring, that is

$$
\left(m_{1}(G) z_{1}^{l} z_{2}^{m}, m_{2}(G) z_{1}^{l} z_{2}^{m}, \cdots, m_{N}(G) z_{1}^{l} z_{2}^{m}\right)=\mathbf{F}\left[z_{1}, z_{2}\right]
$$

(iii) given a basic encoder $G_{+}\left(z_{1}, z_{2}\right) \in \mathbf{F}\left[z_{1}, z_{2}\right]^{k \times n}$, in any factorization

$$
G_{+}\left(z_{1}, z_{2}\right)=T_{+}\left(z_{1}, z_{2}\right) \bar{G}_{+}\left(z_{1}, z_{2}\right),
$$

where the matrix $T_{+}\left(z_{1}, z_{2}\right)$ is a greatest left factor (GLF) of $G_{+}\left(z_{1}, z_{2}\right)$ over $\mathbf{F}\left[z_{1}, z_{2}\right], \bar{G}_{+}\left(z_{1}, z_{2}\right)$ is $\ell \mathrm{ZP}$ over $\mathbf{F}\left[z_{1}, z_{2}\right]$.

Proof (i) $\Rightarrow$ (ii): If $G_{+}\left(z_{1}, z_{2}\right) \in \mathbf{F}\left[z_{1}, z_{2}\right]^{k \times n}$ is a basic encoder, $\ell \mathrm{ZP}$ in $\mathrm{F}\left[z_{1}, z_{2}\right]$, any equivalent basic encoder $G\left(z_{1}, z_{2}\right)$ differs from $G_{+}\left(z_{1}, z_{2}\right)$ in a unimodular matrix $U\left(z_{1}, z_{2}\right) \in \mathscr{F}_{ \pm}^{k \times k}$, namely $G=U G_{+}$. As det $U$ is a unit in $\mathscr{F}_{+}$, that is $\operatorname{det} U=z_{1}^{\nu_{1}} z_{2}^{\nu_{2}}$, it follows that the $i$ th maximal order minor of $G$ is obtained from the corresponding $i$ th minor of $G_{+}$as $m_{i}(G)=z_{1}^{\nu_{1}} z_{2}^{\nu_{2}} m_{i}\left(G_{+}\right)$. So, assuming $l=-\nu_{1}$ and $m=-\nu_{2}$, we satisfy both (5.4) and (5.5).

(ii) $\Rightarrow$ (iii): Let $G_{+}$be a basic encoder with elements in $\mathbf{F}\left[z_{1}, z_{2}\right]$, and consider the pair $(l, m)$ such that the maximal order minors $m_{i}\left(G_{+}\right), i=1,2, \cdots, N$, satisfy (5.4) and (5.5). Clearly, $l$ and $m$ are nonpositive integers, and (5.5) implies that $m_{i}\left(G_{+}\right), i=1,2, \cdots, N$, generate in 
$\mathbf{F}\left[z_{1}, z_{2}\right]$ the principal ideal $\left(z_{1}^{-l} z_{2}^{-m}\right)$, that is

$$
\left(m_{1}\left(G_{+}\right), m_{2}\left(G_{+}\right) \cdots m_{N}\left(G_{+}\right)\right)=\left(z_{1}^{-l} z_{2}^{-m}\right) .
$$

By extracting from $G_{+}$a greatest left factor $T_{+}\left(z_{1}, z_{2}\right)$, we obtain $G_{+}=T_{+} \bar{G}_{+}$. As the determinant of a GLF of $G_{+}$is the greatest common divisor of its maximal order minors $m_{i}\left(G_{+}\right), i=1,2, \cdots, N$, then $\operatorname{det} T_{+}\left(z_{1}, z_{2}\right)=$ $z_{1}^{-l} z_{2}^{-m}$.

Obviously, the maximal order minors of $\bar{G}_{+}$are $m_{1}\left(G_{+}\right) z_{1}^{l} z_{2}^{m}, m_{2}\left(G_{+}\right) z_{1}^{l} z_{2}^{m}, \cdots, m_{N}\left(G_{+}\right) z_{1}^{l} z_{2}^{m}$, and therefore $\bar{G}_{+}$is $/ \mathrm{ZP}$ in $\mathrm{F}\left[z_{1}, z_{2}\right]$.

(iii) $\Rightarrow$ (i): It is immediate that $G_{+}$and $\bar{G}_{+}$are equivalent encoders of $\mathscr{C}$. Being $\ell \mathrm{ZP}$ in $\mathrm{F}\left[z_{1}, z_{2}\right], \bar{G}_{+}$is $\ell \mathrm{ZP}$ also in $\mathscr{F}_{ \pm}$and therefore basic.

Example 4: Let $\mathbf{F}=G F(2)$. The following encoder

$$
G\left(z_{1}, z_{2}\right)=\left[\begin{array}{ccc}
0 & z_{1}+1 & z_{2} \\
1 & 0 & 0
\end{array}\right]
$$

has maximal order minors $z_{1}+1, z_{2}$ and 0 . Since $z_{2}$ is a unit in $\mathscr{F}_{ \pm}, G$ is $\ell \mathrm{ZP}$ in $\mathscr{F}_{ \pm}$and the code $\mathscr{C}$ generated by $G$ is basic. Neither $G$, nor (by the above proposition) any other equivalent encoder of $\mathscr{C}$, is $\ell \mathbf{Z P}$ in $\mathbf{F}\left[z_{1}, z_{2}\right]$.

Using a basic code $\mathscr{E}$ with the aforementioned properties in a transmission system, requires that we first design an encoder $G$ that is $\ell \mathrm{ZP}$ in $\mathrm{F}\left[z_{1}, z_{2}\right]$, and then compute a decoder $G^{-1}$ with elements in $\mathbf{F}\left[z_{1}, z_{2}\right]$. Correspondingly, two finite memory realizations for both $G$ and $G^{-1}$ have to be constructed, by resorting to 2-D realization algorithms available in the literature [21].

Most of the computational effort of the above procedure is devoted to obtaining the transfer matrix of the decoder, and to realizing it as a state model. On the other hand, when relaxing the requirement that $G^{-1}$ has to be a polynomial matrix, a considerable simplification is achieved by resorting to the inverse system technique. Actually, if $\Sigma=\left(A_{1}, A_{2}, B_{1}, B_{2}, C, D\right)$ is a realization of $G$, then $D=G(0,0)$ is right invertible, and for each right inverse of $D$, the corresponding inverse system

$$
\begin{aligned}
\Sigma^{-1}\left(D^{-1}\right)= & \left(A_{1}-C D^{-1} B_{1}, A_{2}-C D^{-1} B_{2},\right. \\
& \left.D^{-1} B_{1}, D^{-1} B_{2},-C D^{-1}, D^{-1}\right)
\end{aligned}
$$

is a realization of a proper rational inverse of $G$. So, an interesting question is to investigate what conditions on $\Sigma$ and $G$ guarantee that the inverse system $\Sigma^{-1}\left(D^{-1}\right)$ is finite memory, and therefore realizes a decoder of $\mathscr{C}$. The following proposition shows how the fact that $\Sigma^{-1}\left(D^{-1}\right)$ inherits the finite memory property of $\Sigma$ only depends on the encoder $G$ and possibly on the constant matrix $D^{-1}$, whereas the particular structure of the state-space realization does not play any role.

Proposition 5.2 [22]-[23]: Let $\Sigma=\left(A_{1}, A_{2}, B_{1}\right.$, $\left.B_{2}, C, D\right)$ be a finite memory 2-D system which realizes a $k \times n$ encoder $G\left(z_{1}, z_{2}\right), \ell \mathrm{ZP}$ over $\mathrm{F}\left[z_{1}, z_{2}\right]$.

For every right inverse $D^{-1}$ of $D$, the following statements are equivalent:

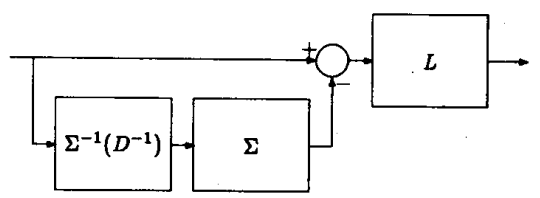

Fig. 4. Syndrome decoder realization.

(i) the inverse system $\Sigma^{-1}\left(D^{-1}\right)$ is finite memory;

(ii) $G\left(z_{1}, z_{2}\right) D^{-1}$ is a unimodular matrix in $\mathbf{F}\left[z_{1}, z_{2}\right]$;

(iii) $G$ can be row bordered into an $n \times n$ matrix

$$
V\left(z_{1}, z_{2}\right)=\left[\begin{array}{c}
G\left(z_{1}, z_{2}\right) \\
K
\end{array}\right]
$$

unimodular in $\mathrm{F}\left[z_{1}, z_{2}\right]$, by any constant full-row rank $(n-k) \times n$ matrix $K$ such that $K D^{-1}=0$.

When $\mathscr{C}$ admits an encoder $G$ that satisfies the above equivalent conditions, the inverse system technique can be applied to any other basic encoder $\tilde{G}$ of $\mathscr{C}, \ell \mathrm{ZP}$ in F $\left[z_{1}, z_{2}\right]$. In fact, by Lemma A.6, $G$ and $\tilde{G}$ differ in an $\mathrm{F}\left[z_{1}, z_{2}\right]$-unimodular matrix $V\left(z_{1}, z_{2}\right)$, that is $\tilde{G}=V G$. Therefore, if $G$ meets condition (ii) of the above proposition,

$$
\tilde{G}\left(z_{1}, z_{2}\right) \tilde{D}^{-1}=\left[V\left(z_{1}, z_{2}\right) G\left(z_{1}, z_{2}\right)\right]\left[D^{-1} V(0,0)^{-1}\right]
$$

is unimodular too.

Before concluding this section, we aim to mention the problem of finding, and realizing through a finite memory system, a syndrome decoder of a given encoder $G, \ell \mathrm{ZP}$ in $\mathrm{F}\left[z_{1}, z_{2}\right]$. In the general case, this requires the construction of a $\ell \mathrm{ZP}$ polynomial matrix $H^{T}\left(z_{1}, z_{2}\right)$, of suitable dimensions, such that $G H^{T}=0$, and the implementation of a finite memory realization of it.

When the encoder $G$ fulfills the equivalent requirements of Proposition 5.2, the problem becomes considerably simpler. Actually, let $G^{-1}\left(z_{1}, z_{2}\right)$ denote the decoder realized by the inverse system $\Sigma^{-1}\left(D^{-1}\right)$ and $\left[D^{-1} L\right]$ the inverse matrix of $\left[\begin{array}{l}D \\ K\end{array}\right], K$ as in (iii) of Proposition 5.2. It is quite easy to prove that the polynomial matrix

$$
H^{T}\left(z_{1}, z_{2}\right):=\left[I-G^{-1}\left(z_{1}, z_{2}\right) G\left(z_{1}, z_{2}\right)\right] L
$$

is a syndrome decoder of $\mathscr{E}(G)$. Moreover, the block scheme of Fig. 4 immediately suggests how to obtain from $\Sigma$ and $\Sigma^{-1}\left(D^{-1}\right)$ the following finite memory realization of $H^{T}$ :

$$
\begin{gathered}
\Sigma_{H^{T}}=\left(\left[\begin{array}{cc}
A_{1}-C D^{-1} B_{1} & -C D^{-1} B_{1} \\
0 & A_{1}
\end{array}\right],\right. \\
{\left[\begin{array}{cc}
A_{2}-C D^{-1} B_{2} & -C D^{-1} B_{2} \\
0 & A_{2}
\end{array}\right],} \\
{\left[\begin{array}{cc}
-D^{-1} B_{1} & \left.-D^{-1} B_{1}\right],\left[\begin{array}{ll}
-D^{-1} B_{2} & -D^{-1} B_{2}
\end{array}\right], \\
\left.\left[\begin{array}{c}
C D^{-1} D H \\
-C H
\end{array}\right],\left[\left(I-D^{-1} D\right) H\right]\right) .
\end{array}\right.}
\end{gathered}
$$




\section{Lemma A.1:}

(i) Let $G\left(z_{1}, z_{2}\right)$ be a $k \times n /$ FP matrix with elements in $\mathscr{F}_{ \pm}$. If $\mathbf{v} \in \mathscr{F}_{ \pm}^{-1 \times n}$ is a linear combination over $\mathbf{F}\left(z_{1}, z_{2}\right)$ of the rows of $G\left(z_{1}, z_{2}\right)$, that is

$$
\mathbf{v}=\mathbf{a} G, \quad \mathbf{a} \in \mathbf{F}\left(z_{1}, z_{2}\right)^{1 \times k}
$$

then a can be chosen in $\mathscr{F}_{ \pm}^{1 \times k}$.

(ii) The same statement holds when $\mathscr{F}_{ \pm}$is replaced by

Proof: (i) Since $G$ is $\ell$ FP, there exist [7] two polynomials $h\left(z_{1}\right) \in \mathbf{F}\left[z_{1}, z_{1}^{-1}\right]$ and $k\left(z_{2}\right) \in \mathbf{F}\left[z_{2}, z_{2}^{-1}\right]$, and two $L$-polynomial matrices $X\left(z_{1}, z_{2}\right)$ and $Y\left(z_{1}, z_{2}\right)$, such that

$$
G X=h\left(z_{1}\right) I_{k} \text { and } G Y=k\left(z_{2}\right) I_{k} .
$$

It entails no loss of generality supposing that the row vector a has irreducible entries, $a_{i}$. So, letting $\beta_{0}$ the least common multiple (LCM) of the denominators of $a_{i},(\mathrm{~A} 1)$ can be rewritten as

$$
\beta_{0} \mathbf{v}=\left[\begin{array}{llll}
\beta_{1} & \cdots & \beta_{k}
\end{array}\right] G \quad \beta_{i} \in \mathscr{F}_{ \pm}, i=1,2 \cdots .
$$

Postmultiplying both members of (A3) by $X\left(z_{1}, z_{2}\right)$ and $Y\left(z_{1}, z_{2}\right)$, we obtain

$$
\begin{aligned}
& \beta_{0} \mathbf{v} X=\left[\begin{array}{lll}
\beta_{1} & \cdots & \beta_{k}
\end{array}\right] G X=\left[\begin{array}{lll}
\beta_{1} & \cdots & \beta_{k}
\end{array}\right] h\left(z_{1}\right) \\
& \beta_{0} \mathbf{v} Y=\left[\begin{array}{lll}
\beta_{1} & \cdots & \beta_{k}
\end{array}\right] G Y=\left[\begin{array}{lll}
\beta_{1} & \cdots & \beta_{k}
\end{array}\right] k\left(z_{2}\right) .
\end{aligned}
$$

As $\beta_{0}, \beta_{1}, \cdots, \beta_{k}$ have no common factors, it follows that $\beta_{0}\left(z_{1}, z_{2}\right) \mid h\left(z_{1}\right)$ and $\beta_{0}\left(z_{1}, z_{2}\right) \mid k\left(z_{2}\right)$, and therefore $\beta_{0}\left(z_{1}, z_{2}\right)$ is a unit in $\mathscr{F}_{ \pm}$.

(ii) Obvious.

Corollary A.2: Let $G\left(z_{1}, z_{2}\right)$ be in $\mathscr{F}_{+}^{k \times n}$, with row rank $\bar{k}$ over $\mathbf{F}\left(z_{1}, z_{2}\right)$. There exist two $L$-polynomial matrices, $\bar{G}\left(z_{1}, z_{2}\right), k \times$ $n \ell \mathrm{FP}$, and $T\left(z_{1}, z_{2}\right), k \times \bar{k}$ with full-column rank, such that

$$
G\left(z_{1}, z_{2}\right)=T\left(z_{1}, z_{2}\right) \bar{G}\left(z_{1}, z_{2}\right) \text {. }
$$

Proof: Let $G^{\prime}\left(z_{1}, z_{2}\right)$ be a matrix obtained by selecting in $G\left(z_{1}, z_{2}\right) \bar{k}$ rows linearly independent over $\mathbf{F}\left(z_{1}, z_{2}\right)$, and $Q\left(z_{1}, z_{2}\right)$ a GLF of $G^{\prime}\left(z_{1}, z_{2}\right)$. Then $G^{\prime}=Q \bar{G}$. Every row in $G$ is a linear combination over $\mathrm{F}\left(z_{1}, z_{2}\right)$ of the rows of $\bar{G}\left(z_{1}, z_{2}\right)$, and, by Lemma $\mathrm{A} 1$, the coefficients of the combination can be chosen in $\mathscr{F}_{ \pm}$.

Therefore $G=T \bar{G}, T\left(z_{1}, z_{2}\right)$ being the $k \times \bar{k}$ matrix of the combinators. As rank $G$ is $\bar{k}$, rank $T$ cannot be less than $\bar{k}$.

Proposition A.3: Let $G\left(z_{1}, z_{2}\right)$ be in $\mathscr{F}^{k \times n}, k \leq n$. $G$ has an $L$-polynomial right inverse if and only if $G$ is $\ell \mathrm{ZP}$.

Proof: If $G$ is $\ell \mathrm{ZP}$, the ideal generated by its maximal order minors $m_{i}(G), i=1,2, \cdots,\left(\begin{array}{l}n \\ k\end{array}\right)$, coincides with the whole ring $\mathscr{F}_{ \pm}$. If follows that there exists $\alpha_{i} \in \mathscr{F}_{ \pm}, i=1,2, \cdots,\left(\begin{array}{l}n \\ k\end{array}\right)$, such that $\sum_{i} \alpha_{i} m_{i}(G)=1$

Consider the identity, $m_{i}(G) I_{k}=G S_{i} \operatorname{Adj}\left(G S_{i}\right)$, where $S_{i}$ denotes the selection matrix corresponding to the minor $m_{i}(G)$. Then we have

$$
\begin{aligned}
I_{k} & =\sum_{i} \alpha_{i} m_{i}(G) I_{k} \\
& =G\left(z_{1}, z_{2}\right)\left[\sum_{i} \alpha_{i}\left(z_{1}, z_{2}\right) S_{i} \operatorname{Adj}\left(G\left(z_{1}, z_{2}\right) S_{i}\right)\right] .
\end{aligned}
$$

Clearly, the $L$-polynomial matrix $K\left(z_{1}, z_{2}\right):=$ $\sum_{i} \alpha_{i}\left(z_{1}, z_{2}\right) S_{i} \operatorname{Adj}\left(G\left(z_{1}, z_{2}\right) S_{i}\right)$ is a right inverse of $G$.

The converse is a direct consequence of the Binet-Cauchy formula.
Proposition A.4: Let $G\left(z_{1}, z_{2}\right) \in \mathscr{F}_{ \pm}^{k \times n}$ and $H^{T}\left(z_{1}, z_{2}\right) \in$ $\mathscr{F}_{ \pm}^{n \times(n-k)}$ be $\ell \mathrm{FP}$ and $r \mathrm{FP}$ matrices, respectively, satisfying $\mathrm{GH}^{ \pm}=0$.

The corresponding maximal order minors of $G$ and $H^{T}$ are equal, modulo a unit of the ring $\mathscr{F}_{ \pm}$.

Proof: Since $G$ is $\ell$ FP, there exist [5] two matrices, $X_{1}$ and $X_{2}$, with elements in $\mathscr{F}_{ \pm}$, and two polynomials, $g_{1}\left(z_{1}\right) \in$ $\mathbf{F}\left[z_{1}, z_{1}^{-1}\right]$ and $g_{2}\left(z_{2}\right) \in \mathbf{F}\left[z_{2}, z_{2}^{-1}\right]$, such that

$$
G_{1} X_{1}=g_{1}\left(z_{1}\right) I_{k} \text { and } G_{2} X_{2}=g_{2}\left(z_{2}\right) I_{k} .
$$

Consider for instance $m_{1}(G)$, the maximal order minor of $G$ corresponding to the selection of the first $k$ columns of $G$. Complete $G$ into a square matrix by resorting to a $(n-k) \times n$ matrix, whose columns are all zero except for the last $n-k$, which constitute the identity matrix. Thus

$$
\left[\begin{array}{ccc} 
& G & \\
0 & & I_{n-k}
\end{array}\right]\left[X_{1} \mid H^{T}\right]=\left[\begin{array}{cc}
g_{1}\left(z_{1}\right) I_{k} & 0 \\
Q & M_{1}\left(H^{T}\right)
\end{array}\right]
$$

where $M_{1}\left(H^{T}\right)$ is the $(n-k) \times(n-k)$ submatrix of $H^{T}$ obtained by selecting the last $n-k$ rows. Assuming $R_{1}\left(z_{1}, z_{2}\right):=$ $\left[\begin{array}{ll}X_{1} & H^{T}\end{array}\right]$ and $\mu_{1}:=\operatorname{det} M_{1}\left(H^{T}\right)$, we get

$$
m_{1}(G) \text { det } R_{1}=\left(g_{1}\left(z_{1}\right)\right)^{k} \mu_{1}\left(H^{T}\right) \text {. }
$$

Now, replace $X_{2}$ with $X_{1}$ in (A5) and let $R_{2}:=\left[X_{2} H^{T}\right]$. We obtain

$$
m_{1}(G) \operatorname{det} R_{2}=\left[g_{2}\left(z_{2}\right)\right]^{k} \mu_{1}\left(H^{T}\right) .
$$

So, $m_{1}(G) \mid\left[g_{1}\left(z_{1}\right)\right]^{k} \mu_{1}\left(H^{T}\right)$ and $m_{1}(G) \mid\left[g_{2}\left(z_{2}\right)\right]^{k} \mu_{1}\left(H^{T}\right)$. Since $\left[g_{1}\left(z_{1}\right)\right]^{k}$ and $\left[g_{2}\left(z_{2}\right)\right]^{k}$ are coprime, then

$$
\left.m_{1}(G)\right|_{\mu_{1}}\left(H^{T}\right) .
$$

Dually, as $H^{T}$ is $r \mathrm{FP}$, there exist two matrices, $Y_{1}$ and $Y_{2}$, with elements in $\mathscr{F}_{ \pm}$, and two polynomials, $h_{1}\left(z_{1}\right) \in \mathbf{F}\left[z_{1}, z_{1}^{-1}\right]$ and $h_{2}\left(z_{2}\right) \in \mathbf{F}\left[z_{2}, z_{2}^{-1}\right]$, such that

$$
Y_{1} H^{T}=h_{1}\left(z_{1}\right) I_{n-k} \text { and } Y_{2} H^{T}=h_{2}\left(z_{2}\right) I_{n-k} .
$$

We can proceed as before, getting

$$
\left[\begin{array}{l}
G \\
Y_{1}
\end{array}\right]\left[\begin{array}{cc}
I_{k} & \\
& H^{T} \\
0 &
\end{array}\right]=\left[\begin{array}{cc}
M_{1}(G) & 0 \\
T & h_{1}\left(z_{1}\right) I_{n-k}
\end{array}\right]
$$

where $M_{1}(G)$ is the $k \times k$ submatrix of $G$ obtained by selecting its first $k$ columns. Assuming $S_{1}:=\left[\begin{array}{l}G \\ Y_{1}\end{array}\right]$, we get $\operatorname{det} S_{1} \mu_{1}\left(H^{T}\right)$ $=m_{1}(G)\left[h_{1}\left(z_{1}\right)\right]^{n-k}$, and, analogously, $\operatorname{det} S_{2} \mu_{1}\left(H^{T}\right)=$ $m_{1}(G)\left[h_{2}\left(z_{2}\right)\right]^{n-k}$, where $S_{2}:=\left[\begin{array}{c}G \\ Y_{2}\end{array}\right]$.

Therefore, $\mu_{1}\left(H^{T}\right)$ is a common factor of $m_{1}(G)\left[h_{1}\left(z_{1}\right)\right]^{n-\kappa}$ and $m_{1}(G)\left[h_{2}\left(z_{2}\right)\right]^{n-k}$, and then

$$
\mu_{1}\left(H^{T}\right) \mid m_{1}(G) .
$$

(A6) and (A7) together imply that $m_{1}(G)$ and $\mu_{1}\left(H^{T}\right)$ differ in a unit of $\mathscr{F}_{+}$.

Similarly, we can show that the same result holds for any other pair of corresponding minors in $G$ and in $H^{T}$.

Lemma A.5: Let $H^{T}\left(z_{1}, z_{2}\right) \in \mathscr{F}_{ \pm}^{n \times(n-k)}$. The map $H^{T}: \mathscr{F}_{ \pm}^{n}$ $\rightarrow \mathscr{F}_{+}^{n-k}: \mathrm{w} \mapsto \mathrm{w} H^{T}$ is onto if and only $H^{T}$ is $r \mathrm{ZP}$.

Proof: Assume that $H^{T}$ is onto. Then there exist

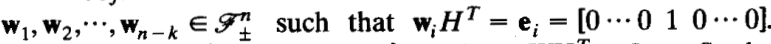
Letting $W=\operatorname{col}\left\{w_{1}, w_{2}, \cdots, w_{n-k}\right\}$, we have $W H^{T}=I_{n-k}$. So, by Proposition A.3, $H^{T}\left(z_{1}, z_{2}\right)$ is $r \mathrm{ZP}$. 
Conversely, if $H^{T}$ is $r \mathrm{ZP}$, it admits a left inverse $W \in$ $\mathscr{F}_{ \pm}^{(n-k) \times n}$. So, for every $\mathbf{p} \in \mathscr{F}_{ \pm}^{n-k}$ we have $\mathbf{p}=\mathbf{p} I_{n-k}=$ $(\mathrm{pW}) H^{T}$, which implies that $\mathbf{p}$ is the image, under $H^{T}$, of an $L$-polynomial vector.

Lemma A.6: Suppose that $\bar{G}_{1}\left(z_{1}, z_{2}\right)$ and $\bar{G}_{2}\left(z_{1}, z_{2}\right)$ are $k \times n$ polynomial matrices, $\ell \mathrm{FP}$ in $\mathrm{F}\left[z_{1}, z_{2}\right]$. If there exists an $\mathscr{F}_{ \pm}$-unimodular matrix $U\left(z_{1}, z_{2}\right)$ such that

then

$$
\bar{G}_{1}\left(z_{1}, z_{2}\right)=U\left(z_{1}, z_{2}\right) \bar{G}_{2}\left(z_{1}, z_{2}\right)^{\prime}
$$

$$
\bar{G}_{1}\left(z_{1}, z_{2}\right)=V\left(z_{1}, z_{2}\right) \bar{G}_{2}\left(z_{1}, z_{2}\right)
$$

for some $\mathbb{N}\left[z_{1}, z_{2}\right]$-unimodular matrix $V$.

Proof: By assumption (A8), every row of $\bar{G}_{1}$ is a linear combination over $\mathbf{F}\left(z_{1}, z_{2}\right)$ of the rows of $\bar{G}_{2}$. By Lemma A.1, there exists a matrix $V\left(z_{1}, z_{2}\right) \in \mathbf{F}\left[z_{1}, z_{2}\right]^{k \times k}$ such that $\bar{G}_{1}=$ $V \bar{G}_{2}$. Since $\bar{G}_{1}$ is $/ \mathbf{F P}$, det $V$ is a nonzero constant and $V$ is $\mathrm{F}\left[z_{1}, z_{2}\right]$-unimodular.

\section{REFERENCES}

[1] G. D. Forney, Jr., "Convolutional codes I: Algebraic structure," IEEE Trans. Inform. Theory, vol. IT-16, pp. 720-738, November 1970.

[2] IEEE Trans. Inform. Theory, vol. IT-19, pp. 512-518; May 1973.

[3] - " Minimal bases of rational vector spaces, with applications to multivariable linear systems," SLAM J. Contr., vol. 13, pp. 493-520, May 1975.

[4] B. C. Lévy, " $2 \mathrm{D}$ polynomial and rational matrices, and their applications for the modeling of $2 \mathrm{D}$ dynamical systems," Tech Rep. M735-11, Stanford Electron. Lab., Standford Univ., 1981.

[5] D. C. Youla and P. F. Pickel, "The Quillen-Suslin theorem," IEEE Trans. Circ. Syst., vol. CAS-31, pp. 513-518, June 1984.

[6] D. C. Youla and G. Gnavi, "Notes on $n$-dimensional system theory," IEEE Trans. Circ. Syst., vol. CAS-26, pp. 105-111, February 1979.

[7] M. Morf, B. C. Lévy, S. Y. Kung, and T. Kailath, "New results in 2D systems theory: Part I and II," Proc. IEEE, vol. 65, pp. 861-872 and 945-961, June 1977.
[8] J. C. Willems, "Models for dynamics," Dynam. Rep. vol. 2, pp. 171-267, 1989.

[9] P. Rocha, "Structure and representation of 2D systems," Ph.D. dissertation, Rijksuniversiteit, Groningen, 1990.

[10] P. Rocha and J. C. Willems, "Controllability of 2D systems," IEEE Trans. Automat. Control, vol. AC-36, pp. 413-423, April 1991.

[11] J. C. Willems, "Paradigms and puzzles in the theory of dynamical systems," IEEE Trans. Automat. Control, vol. AC-36, pp. 259-294, March 1991.

[12] E. Fornasini and S. Zampieri, "A note on the state space realization of 2D FIR transfer functions," Syst. Contr. Letters, vol. 16, pp. 17-22, 1990.

[13] M. Bisiacco, "State and output feedback stabilizability of 2D systems," IEEE Trans. Circ. Syst., vol. CAS-32, pp. 1246-54, December 1985.

[14] E. Fornasini and G. Marchesini, "Properties of pairs of matrices and state models for 2D systems, Pt. I: State dynamics and geometry of the pairs; Pt. II: Models structure and realization problems," in Multivariate Analysis: Future Directions, C. R. Rao, Ed., North Holland Series in Probability and Statistics, vol. 5. Amsterdam, The Netherlands: North Holland, 1993, pp. 131-180.

[15] Ph. Piret, Convolutional Codes. Cambridge, MA: MIT, 1981.

[16] S. Lang, Algebra. Reading, MA: Addison-Wesley, 1967.

[17] E. Fornasini, P. Rocha, and S. Zampieri, "State space realization of 2D finite dimensional behaviours," SLAM J. Contr. Optim., vol. 31, no. 6, pp. 1502-1517, Nov. 1993.

[18] E. Fornasini and M. E. Valcher, "An algebraic approach to 2D convolutional codes. Pt. I," Tech. Rep., 1992.

[19] W. Greub, Linear algebra. New York: Springer-Verlag, 1975.

[20] E. Fornasini and G. Marchesini, "State space realization of twodimensional filters," IEEE Trans. Automat. Contr., vol. AC-21, pp. 484-492, August 1976.

[21] M. Bisiacco, E. Fornasini, and Marchesini, "Dynamic regulation of 2D systems: A state-space approach," Lin. Alg. Appl, vol. 122/124, pp. 195-218, 1989.

[22] E. Fornasini and M. E. Valcher, "Polynomial inverses of 2D transfer matrices and finite memory realizations via inverse systems," Multidim. Syst. Signal Proc., vol. 4, pp. 269-294, 1993.

[23] E. Fornasini, G. Marchesini, and M. E. Valcher, "On the structure of finite memory and separable 2D systems," Automatica, vol. 30, no. 2, Feb. 1994 . 